\title{
On 2-connected hypergraphs with no long cycles
}

\author{
Zoltán Füredi * \\ Alfréd Rényi Institute of Mathematics \\ Budapest, Hungary \\ z-furedi@illinois.edu
}

\author{
Alexandr Kostochka ${ }^{\dagger}$ \\ Department of Mathematics \\ University of Illinois at Urbana-Champaign \\ Urbana, Illinois, U.S.A. \\ kostochk@math.uiuc.edu
}

\author{
Ruth Luo $\ddagger$ \\ Department of Mathematics \\ University of California, San Diego \\ La Jolla, California, U.S.A. \\ ruluo@ucsd.edu
}

Submitted: Jan 31, 2019; Accepted: Oct 1, 2019; Published: Nov 8, 2019

(C) The authors. Released under the CC BY license (International 4.0).

\begin{abstract}
We give an upper bound for the maximum number of edges in an $n$-vertex 2connected $r$-uniform hypergraph with no Berge cycle of length $k$ or greater, where $n \geqslant k \geqslant 4 r \geqslant 12$. For $n$ large with respect to $r$ and $k$, this bound is sharp and is significantly stronger than the bound without restrictions on connectivity. It turned out that it is simpler to prove the bound for the broader class of Sperner families where the size of each set is at most $r$. For such families, our bound is sharp for all $n \geqslant k \geqslant r \geqslant 3$.
\end{abstract}

Mathematics Subject Classifications: 05D05, 05C65, 05C38, 05C35

\subsection{Basic definitions}

The upper rank of a hypergraph $\mathcal{H}$ is the size of a largest edge. For brevity, instead of saying "a hypergraph of upper rank $r$ " we will say "an $r^{-}$-graph". When every edge has size $r$, i.e., $\mathcal{H}$ is $r$-uniform, we call $\mathcal{H}$ an "r-graph".

\footnotetext{
*Supported in part by the Hungarian National Research, Development and Innovation Office NKFIH grant KH-130371, and by the Simons Foundation Collaboration Grant 317487.

${ }^{\dagger}$ Supported in part by NSF grant DMS-1600592 and grants 18-01-00353A and 19-01-00682 of the Russian Foundation for Basic Research.

${ }^{\ddagger}$ Supported in part by NSF grant DMS-1902808 and by Award RB17164 of the Research Board of the University of Illinois at Urbana-Champaign.
} 
A hypergraph $\mathcal{H}$ is Sperner if no edge of $\mathcal{H}$ is contained in another edge. In particular, a Sperner hypergraph has no multiple edges, and all simple uniform hypergraphs are Sperner.

Definition 1. A Berge cycle of length $\ell$ in a hypergraph is a set of $\ell$ distinct vertices $\left\{v_{1}, \ldots, v_{\ell}\right\}$ and $\ell$ distinct edges $\left\{e_{1}, \ldots, e_{\ell}\right\}$ such that $\left\{v_{i}, v_{i+1}\right\} \subseteq e_{i}$ with indices taken modulo $\ell$. The vertices $\left\{v_{1}, \ldots, v_{\ell}\right\}$ are called base vertices of the Berge cycle.

A Berge path of length $\ell$ in a hypergraph is a set of $\ell+1$ distinct vertices $\left\{v_{1}, \ldots, v_{\ell+1}\right\}$ and $\ell$ distinct hyperedges $\left\{e_{1}, \ldots, e_{\ell}\right\}$ such that $\left\{v_{i}, v_{i+1}\right\} \subseteq e_{i}$ for all $1 \leqslant i \leqslant \ell$. The vertices $\left\{v_{1}, \ldots, v_{\ell+1}\right\}$ are called base vertices of the Berge path.

Definition 2. The incidence bigraph of a hypergraph $\mathcal{H}$ is the bipartite graph $I(\mathcal{H})=$ $(A, Y ; E)$ such that $A=E(\mathcal{H}), Y=V(\mathcal{H})$ and for $a \in A, y \in Y$, ay $\in E(I(\mathcal{H}))$ if and only if $y \in a$ in $\mathcal{H}$.

A cycle $C$ of length $2 \ell$ in $I(\mathcal{H})$ corresponds to a Berge cycle of length $\ell$ in $\mathcal{H}$ with the set of base vertices $C \cap Y$ and the set of edges $C \cap A$. Similarly, a path $P$ of length $2 \ell+1$ (vertices) in $I(\mathcal{H})$ with endpoints in $Y$ corresponds to a Berge path of length $\ell$ in $\mathcal{H}$ with the set of base vertices $P \cap Y$ and the set of edges $C \cap A$.

Definition 3. A hypergraph $\mathcal{H}$ is called 2-connected if its incidence bigraph is $I(\mathcal{H})$ is 2-connected.

A block in a graph $G$ is a maximal connected subgraph $G^{\prime}$ with no cut vertices (of $G^{\prime}$ ).

So $\mathcal{H}$ is 2-connected if it is connected and has neither cut vertex (i.e., a vertex $v \in V(\mathcal{H})$ for which there is a partition of $V(\mathcal{H})=\{v\} \cup V_{1} \cup V_{2},\left|V_{i}\right| \geqslant 1$, such that every edge is contained in either $\{v\} \cup V_{1}$ or $\{v\} \cup V_{2}$ ), nor a cut edge (i.e., an edge $e \in \mathcal{H}$ for which there is a partition of $V(\mathcal{H})=V_{1} \cup V_{2},\left|V_{i}\right| \geqslant 1$, such that every edge $f \neq e$ is contained in either $V_{1}$ or in $V_{2}$ ).

Let $\mathcal{H}$ be a hypergraph and $p$ be an integer. The $p$-shadow, $\partial_{p} \mathcal{H}$, is the collection of the $p$-sets that lie in some edge of $\mathcal{H}$. In particular, we will often consider the 2 -shadow $\partial_{2} \mathcal{H}$ of an $r$-uniform hypergraph $\mathcal{H}$. Each edge $e$ of $\mathcal{H}$ yields in $\partial_{2} \mathcal{H}$ a clique on $|e|$ vertices. Note that if a hypergraph is 2-connected, then its 2-shadow is a 2-connected graph. The converse is not always true.

\subsection{Graphs without long cycles}

The classic Turán-type result on graphs without long cycles is:

Theorem 4 (Erdös and Gallai [1]). Let $k \geqslant 3$ and let $G$ be an n-vertex graph with more than $\frac{1}{2}(k-1)(n-1)$ edges. Then $G$ contains a cycle of length at least $k$.

This bound is sharp for infinitely many $n$ : when $k-2$ divides $n-1$, the circumference of each connected $n$-vertex graph whose blocks (maximal connected subgraphs with no cut vertices) are cliques of order $k-1$ is only $k-1$. 
There have been several alternate proofs and sharpenings of the Erdös-Gallai theorem including results by Woodall [18], Lewin [16], Faudree and Schelp [5, 6], and Kopylov [14]; see [10] for further details.

The strongest version was that of Kopylov who improved the Erdős-Gallai bound for 2-connected graphs. To state the theorem, we first introduce the family of extremal graphs.

Construction 5. Fix $k \geqslant 4, n \geqslant k, \frac{k}{2}>a \geqslant 1$. Define the $n$-vertex graph $H_{n, k, a}$ as follows. The vertex set of $H_{n, k, a}$ is partitioned into three sets $A, B, C$ such that $|A|=a$, $|B|=n-k+a$ and $|C|=k-2 a$ and the edge set of $H_{n, k, a}$ consists of all edges connecting $A$ with $B$ and all edges in $A \cup C$.

Note that when $a \geqslant 2, H_{n, k, a}$ is 2-connected, has no cycle of length $k$ or longer, and $e\left(H_{n, k, a}\right)=\left(\begin{array}{c}k-a \\ 2\end{array}\right)+(n-k+a) a$.

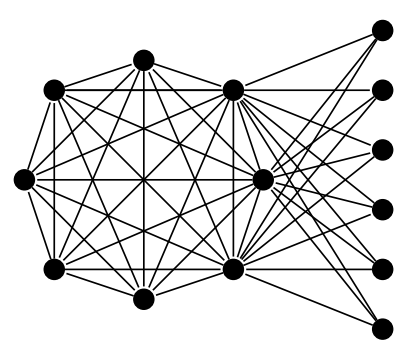

Figure 1: $H_{14,11,3}$

Theorem 6 (Kopylov [14]). Let $n \geqslant k \geqslant 5$ and let $t=\left\lfloor\frac{k-1}{2}\right\rfloor$. If $G$ is a 2-connected n-vertex graph with

$$
e(G)>\max \left\{e\left(H_{n, k, 2}\right), e\left(H_{n, k, t}\right)\right\},
$$

then $G$ has a cycle of length at least $k$.

Furthermore, Kopylov's proof yields that the only sharpness examples are the graphs $G_{n, k, t}$ and $G_{n, k, 2} ;$ see [10] for details.

\subsection{Hypergraphs without long Berge cycles}

Recently, several interesting results were obtained for Berge paths and cycles. Notably, the results depend on the relationship between $k$ and $r$.

Theorem 7 (Györi, Katona, and Lemons [11]). Let $\mathcal{H}$ be an n-vertex $r$-graph with no Berge path of length $k$. If $r \geqslant k \geqslant 3$, then $e(\mathcal{H}) \leqslant \frac{(k-1) n}{r+1}$. If $k>r+1>3$, then $e(\mathcal{H}) \leqslant \frac{n}{k}\left(\begin{array}{l}k \\ r\end{array}\right)$.

Later, the remaining case $k=r+1$ was resolved by Davoodi, Györi, Methuku, and Tompkins [2]. Furthermore, the bounds in Theorem 7 and in [2] are sharp for each $k$ and $r$ for infinitely many $n$. 
Győri, Methuku, Salia, Tompkins, and Vizer [13] proved an asymptotic version of the Erdős-Gallai theorem for Berge paths in connected hypergraphs whenever $r$ is fixed and $n$ and $k$ tend to infinity.

Theorem 8 (Győri, Methuku, Salia, Tompkins, and Vizer [13]). Let $r$ be given. Let $\mathcal{H}_{n, k}$ be a largest $r$-uniform connected n-vertex hypergraph with no Berge path of length $k$. Then

$$
\lim _{k \rightarrow \infty}\left(\lim _{n \rightarrow \infty} \frac{e\left(\mathcal{H}_{n, k}\right)}{k^{r-1} n}\right)=\frac{1}{2^{r-1}(r-1) !} .
$$

For Berge cycles, the exact result for $k \geqslant r+3$ was obtained in [7]:

Theorem 9 (Füredi, Kostochka and Luo [7]). Let $k \geqslant r+3 \geqslant 6$, and let $\mathcal{H}$ be an $n$-vertex $r$-graph with no Berge cycles of length $k$ or longer. Then $e(\mathcal{H}) \leqslant \frac{n-1}{k-2}\left(\begin{array}{c}k-1 \\ r\end{array}\right)$.

This theorem is a hypergraph version of Theorem 4 for $k \geqslant r+3$. The case of $k \leqslant r-1$ was resolved by Kostochka and Luo [15].

Theorem 10 (Kostochka and Luo [15]). Let $k \geqslant 4, r \geqslant k+1$, and let $\mathcal{H}$ be an n-vertex $r$-graph with no Berge cycles of length $k$ or longer. Then $e(\mathcal{H}) \leqslant \frac{(k-1)(n-1)}{r}$.

Recently, Ergemlidze, Győri, Methuku, Salia, Thompkins, and Zamora [4] extended the results to $k \in\{r+1, r+2\}$, and Györi, Lemons, Salia, and Zamora [12] extended the results to $k=r$.

Theorem 11 (Ergemlidze et al. [4]). If $k \geqslant 4$ and $\mathcal{H}$ is an n-vertex $r$-graph with no Berge cycles of length $k$ or longer, then $k=r+1$ and $e(\mathcal{H}) \leqslant n-1$, or $k=r+2$ and $e(\mathcal{H}) \leqslant \frac{n-1}{k-2}\left(\begin{array}{c}k-1 \\ r\end{array}\right)$.

Theorem 12 (Györi et al. [12]). If $r \geqslant 3$ and $\mathcal{H}$ is an n-vertex $r$-graph with no Berge cycles of length $r$ or longer, then $e(\mathcal{H}) \leqslant \max \left\{\left\lfloor\frac{n-1}{r}\right\rfloor(r-1), n-r+1\right\}$.

Theorems 9 and 10 are sharp for each $k$ and $r$ for infinitely many $n$. Furthermore, the present authors also proved in [8] exact bounds for all $n$ when $k \geqslant r+4$.

For $r \geqslant k+1$, bounds for 2-connected hypergraphs stronger than for the general case were found in [15], although they are not known to be sharp.

Theorem 13 (Kostochka and Luo [15]). Let $k \geqslant 4, r \geqslant k+1$, and let $\mathcal{H}$ be an $n$ vertex 2 -connected, $r$-uniform hypergraph with no Berge cycle of length $k$ or longer. Then $e(\mathcal{H}) \leqslant \max \left\{k-1, \frac{k}{2 r-k+2}(n-1)\right\}$.

In this paper, we find sharp bounds on the maximum number of edges in a 2-connected $r$-uniform hypergraph without Berge cycle of length $k$ or longer in the case $k \geqslant 4 r$ for $n>k 2^{r}$. We do this by proving a more general sharp bound for Sperner $r^{-}$-graphs. 


\section{Results}

\subsection{Results on 2-connected hypergraphs with no long Berge cycles}

Our goal is to prove a version of Kopylov's theorem for hypergraphs, i.e., to find the maximum number of edges in a 2-connected hypergraph with no Berge cycle of length $k$ or greater.

Define

$$
f(n, k, r, a):=\left(\begin{array}{c}
k-a \\
\min \left\{r,\left\lfloor\frac{k-a}{2}\right\rfloor\right\}
\end{array}\right)+(n-k+a)\left(\begin{array}{c}
a \\
\min \{r-1,\lfloor a / 2\rfloor\}
\end{array}\right) .
$$

Also define

$$
f^{*}(n, k, r, a):=\left(\begin{array}{c}
k-a \\
r
\end{array}\right)+(n-k+a)\left(\begin{array}{c}
a \\
r-1
\end{array}\right) .
$$

Note that $f(n, k, r, a)=f^{*}(n, k, r, a)$ whenever $r \leqslant\lfloor(k-a) / 2\rfloor$ and $r-1 \leqslant\lfloor a / 2\rfloor$. Our main result is:

Theorem 14. Let $n \geqslant k \geqslant r \geqslant 3$. If $\mathcal{H}$ is an $n$-vertex Sperner 2-connected $r^{-}$hypergraph with no Berge cycle of length $k$ or longer, then $e(\mathcal{H}) \leqslant \max \{f(n, k, r,\lfloor(k-$ $1) / 2\rfloor), f(n, k, r, 2)\}$.

This bound is sharp. To see this, we construct a series of hypergraphs (not necessarily uniform). The following can be viewed as a hypergraph version of Construction 5 .

Construction 15. For $n \geqslant k \geqslant r, 1 \leqslant a \leqslant\lfloor(k-1) / 2\rfloor$, let $\mathcal{H}_{n, k, r, a}$ be the hypergraph with vertex set $A \cup B \cup C$ such that $|A|=k-2 a,|B|=a,|C|=n-(k-a)$. The edge set of $\mathcal{H}_{n, k, r, a}$ is the family

$\{e \subseteq A \cup B:|e|=\min \{r,\lfloor(k-a) / 2\rfloor\}\} \cup\left\{c \cup e^{\prime}: c \in C, e^{\prime} \subseteq B,\left|e^{\prime}\right|=\min \{r-1,\lfloor a / 2\rfloor\}\right\}$.

For $a \geqslant 2, \mathcal{H}_{n, k, r, a}$ is 2-connected and contains no Berge cycle of length $k$ or longer. We have that $\left|E\left(\mathcal{H}_{n, k, r, a}\right)\right|=f(n, k, r, a)$, which is maximized when $a=\lfloor(k-1) / 2\rfloor$ or $a=2$ by the convexity of $f$ (as a function of $a$, see Claim 67 in the appendix). Furthermore, when $r \leqslant\lfloor(k-a) / 2\rfloor$ and $r-1 \leqslant\lfloor a / 2\rfloor, \mathcal{H}_{n, k, r, a}$ is $r$-uniform with $f^{*}(n, k, r, a)$ edges.

For integers $k \geqslant r$, let $n_{k, r}$ be the smallest positive integer $n$ such that $f(n, k, r,\lfloor(k-$ $1) / 2\rfloor) \geqslant f(n, k, r, 2)$. Asymptotically $n_{k, r}$ is about $2^{r-1} k / r$. Then as a corollary of Theorem 14 we obtain the following result for $r$-graphs.

Theorem 16. Let $n \geqslant n_{k, r} \geqslant k \geqslant 4 r \geqslant 12$. If $\mathcal{H}$ is an n-vertex 2 -connected $r$-graph with no Berge cycle of length $k$ or longer, then $e(\mathcal{H}) \leqslant f(n, k, r,\lfloor(k-1) / 2\rfloor)=f^{*}(n, k, r,\lfloor(k-$ 1)/2」).

For $n$ large, this bound is almost $2^{r-1} / r$ stronger than the (exact) bound in Theorem 9 with no restriction on connectivity. Again we have sharpness example $\mathcal{H}_{n, k, r,\lfloor(k-1) / 2\rfloor}$. 


\subsection{Connected hypergraphs with no long Berge path}

We also obtain a result for connected graphs with no Berge path of length $k$.

Theorem 17. Let $n \geqslant k \geqslant r \geqslant 3$. If $\mathcal{H}$ is an $n$-vertex Sperner connected $r^{-}$-graph with no Berge path of length $k$, then $e(\mathcal{H}) \leqslant \max \{f(n, k, r,\lfloor(k-1) / 2\rfloor), f(n, k, r, 1)\}$.

For integers $k \geqslant r$, let $n_{k, r}^{\prime}$ be the smallest positive integer $n$ such that $f(n, k, r,\lfloor(k-$ $1) / 2\rfloor) \geqslant f(n, k, r, 1)$. Then we obtain the following result for $r$-uniform graphs with no Berge path of length $k$ as a corollary of Corollary 17. This improves Theorem 8.

Theorem 18. Let $n \geqslant n_{k, r}^{\prime} \geqslant k \geqslant 4 r \geqslant 12$. If $\mathcal{H}$ is an $n$-vertex connected $r$-graph with no Berge path of length $k$, then $e(\mathcal{H}) \leqslant f(n, k, r,\lfloor(k-1) / 2\rfloor)=f^{*}(n, k, r,\lfloor(k-1) / 2\rfloor)$.

The family $\mathcal{H}_{n, k, r,\lfloor(k-1) / 2\rfloor}$ again shows sharpness of our bounds.

\section{Proof outline}

The basic idea of the proof is to consider instead of the family of $r$-graphs the larger family of Sperner $r^{-}$-graphs. Then we can in some situations shrink some edges keeping the $r^{-}$-graph Sperner.

We start with a dense Sperner $r^{-}$-graph $\mathcal{H}$. By definition, each edge $e$ in $\mathcal{H}$ yields a clique of order $|e|$ in the 2 -shadow of $\mathcal{H}$. If $\mathcal{H}$ contains a long Berge cycle $C$, then $\partial_{2} \mathcal{H}$ contains a cycle of the same length. However, the converse is not always true. So, our first goal is to reduce $\mathcal{H}$ to a smaller dense Sperner $r^{-}$-graph $\mathcal{H}^{\prime}$ for which we know that the existence of a long cycle in $\partial_{2} \mathcal{H}^{\prime}$ implies the existence of a long cycle in $\mathcal{H}^{\prime}$ itself.

Our second goal is to give an upper bound on the maximum size of a Sperner family of cliques of order at most $r$ in the shadow $\partial_{2} \mathcal{H}^{\prime}$ that does not have long cycles. This automatically yields a bound on $\left|\mathcal{H}^{\prime}\right|$.

We systematically consider incidence graphs of $r^{-}$-graphs instead of the $r^{-}$-graphs themselves, because we find the language of 2-connected bipartite graphs convenient for our goals.

In Section 4, we prove two results for the maximum number of cliques in graphs without long cycles or paths which will later be applied to the 2-shadows of $r^{-}$-graphs. Specifically, we give upper bounds for the size of Sperner families of cliques of size at most $r$ in graphs with bounded circumference and graphs that do not contain long paths between every pair of vertices.

In Sections 5 and 6, we prove that our hypergraph can be reduced to dense subhypergraph with no long cycles in its 2 -shadow. We use the language of incidence bigraphs in Section 5 and the language of hypergraphs in Section 6. In Section 7, we combine the results from Sections 4-6 to prove Theorem 14. Finally, in Section 8 we prove Theorem 17 for Berge paths in connected hypergraphs. 


\section{Sperner cliques in graphs}

A set family $H$ is called Sperner if no element of $H$ is contained in another element of $H$. In particular, every uniform family is Sperner. The classic proof of LYM Inequality yields also the following result.

Theorem 19. Let $H$ be a set of $h$ elements. Let $\mathcal{C}$ be a Sperner family of subsets of $H$ such that $|C| \leqslant r$ for each $C \in \mathcal{C}$. Then $|\mathcal{C}| \leqslant\left(\begin{array}{c}h \\ \min \{r,\lfloor h / 2\rfloor\}\end{array}\right)$.

\subsection{Cliques in graphs with bounded circumference}

In [17], Luo proved an upper bound for the maximum number of cliques in a 2-connected graph with bounded circumference.

Theorem 20 (Luo [17]). Let $n \geqslant k \geqslant 5, r \geqslant 2$ be positive integers. Let $G$ be an n-vertex 2 -connected graph with no cycle of length $k$ or longer. Then the number of copies of $K_{r}$ in $G$ is at most

$$
\max \left\{\left(\begin{array}{c}
k-2 \\
r
\end{array}\right)+(n-k+2)\left(\begin{array}{c}
2 \\
r-1
\end{array}\right),\left(\begin{array}{c}
\lceil(k+1) / 2\rceil \\
r
\end{array}\right)+(n-\lceil(k+1) / 2\rceil)\left(\begin{array}{c}
\lfloor(k-1) / 2\rfloor \\
r-1
\end{array}\right)\right\} .
$$

We will prove a version of Theorem 20 for Sperner families of cliques. Recall

$$
f(n, k, r, a):=\left(\begin{array}{c}
k-a \\
\min \left\{r,\left\lfloor\frac{k-a}{2}\right\rfloor\right\}
\end{array}\right)+(n-k+a)\left(\begin{array}{c}
a \\
\min \{r-1,\lfloor a / 2\rfloor\}
\end{array}\right) .
$$

For fixed positive integers $n \geqslant k \geqslant r, f(n, k, r, a)$ is convex over integers $a$ in $[0,\lfloor(k-$ $1) / 2\rfloor$ ] (see the appendix for a proof). Thus the value of $f(n, k, r, a)$ is maximized at one of the endpoints of the domain. For a graph $G$ and a positive integer $r$, let $N_{\mathrm{Sp}}(G, r)$ denote the maximum size of a Sperner family $\mathcal{C}$ of subsets of $V(G)$ such that for each $C \in \mathcal{C}, G[C]$ is a clique of size at most $r$.

Theorem 21. Let $n \geqslant k \geqslant 5, r \geqslant 2$ be positive integers. Let $G$ be an $n$-vertex 2 -connected graph with no cycle of length $k$ or longer. Then

$$
N_{\mathrm{Sp}}(G, r) \leqslant \max \{f(n, k, r, 2), f(n, k, r,\lfloor(k-1) / 2\rfloor)\} .
$$

To prove Theorem 21, we use a structural theorem by Kopylov for 2-connected graphs without long cycles.

Definition 22. For a positive integer $\alpha$ and a graph $G$, the $\alpha$-disintegration of a graph $G$ is the process of iteratively removing from $G$ the vertices with degree at most $\alpha$ until the resulting graph has minimum degree at least $\alpha+1$ or is empty. This resulting subgraph $H(G, \alpha)$ is called the $(\alpha+1)$-core of $G$. It is well known (and easy) that $H(G, \alpha)$ is unique and does not depend on the order of vertex deletion. If $H(G, \alpha)$ is the empty graph, then we say that $G$ is $\alpha$-disintegrable. 
Theorem 23 (Kopylov [14]). Let $n \geqslant k \geqslant 5$ and let $t=\left\lfloor\frac{k-1}{2}\right\rfloor$. Suppose that $G$ is a 2 -connected $n$-vertex graph with no cycle of length at least $k$.

Then either

(23.1) the t-core $H(G, t)$ is empty, the graph $G$ is $t$-disintegrable; or

(23.2) $|H(G, t)|=s$ for some $t+2 \leqslant s \leqslant k-2$, and $H(G, t)=H(G, k-s)$, i.e., the rest of the vertices can be removed by a $(k-s)$-disintegration.

Proof of Theorem 21. Set $t:=\lfloor(k-1) / 2\rfloor$. Let $G$ be an $n$-vertex 2-connected graph with no cycle of length $k$ or longer. Let $\mathcal{C}$ be a Sperner family of subsets of $V(G)$ that are cliques of size at most $r$ with $|\mathcal{C}|=N_{\mathrm{Sp}}(G, r)$. Apply Theorem 23 to $G$. If (23.1) holds, then every vertex is deleted in the $t$-disintegration. At the time of its deletion, each vertex $v$ has at most $t$ neighbors and by Theorem 19, is contained in at most $\left(\begin{array}{c}t \\ \min \{r-1,[t / 2]\}\end{array}\right)$ cliques of $\mathcal{C}$ (since each clique containing $v$ has at most $r-1$ other vertices). After $n-k+t$ steps in the disintegration process, the remaining $k-t$ vertices contain at most $\left(\begin{array}{c}k-t \\ \min \{\lfloor((k-t) / 2)\rfloor, r\}\end{array}\right)$ elements of $\mathcal{C}$. Therefore $|\mathcal{C}| \leqslant N_{\mathrm{Sp}}(G, r) \leqslant f(n, k, r, t)$.

Now suppose (23.2) holds. Then we consecutively delete vertices of degree at most $k-s$ until we arrive at the core $H(G, t)$ of size $s$. As in the previous case, when deleting a vertex $v$ of degree at most $k-s$, we remove at most $\left(\begin{array}{c}k-s \\ \min \{(k-s) / 2, r-1\}\end{array}\right)$ cliques of $\mathcal{C}$ containing $v$. Since $H(G, t)$ contains at most $\left(\begin{array}{c}s \\ \min \{s / 2, r\}\end{array}\right)=\left(\begin{array}{c}k-(k-s) \\ \min \{(k-(k-s)) / 2\rfloor, r\}\end{array}\right)$ cliques in $\mathcal{C}$, we obtain

$$
|\mathcal{C}|=N_{\mathrm{Sp}}(G, r) \leqslant f(n, k, k-s) \leqslant \max \{f(n, k, r, 2), f(n, k, r, t)\}
$$

The last inequality holds by the convexity of $f$.

\section{$3.2 k$-path connected graphs}

A graph $G$ is $\ell$-hamiltonian if for each linear forest $L$ with $\ell$ edges (and no isolated vertex) on the vertex set $V(G)$ there is a hamiltonian cycle in $G \cup L$ that contains $L$.

A graph $G$ is $k$-path connected if for each pair of vertices $x, y \in V(G), G$ contains an $x, y$-path with $k$ or more vertices. In particular, every $n$-vertex 1-hamiltonian graph is $n$-path connected. The following theorem will be helpful for us.

Theorem 24 (Enomoto [3]). Let $G$ be a 3-connected graph on $n$ vertices such that for every pair of vertices $u, v$ such that $u v \notin E(G), d(u)+d(v) \geqslant t$. Then $G$ is k-path connected where $k=\min \{n, 2 t-1\}$.

Define the function

$$
h_{\mathrm{Sp}}(n, \ell, r, d):=\left(\begin{array}{c}
n-d+\ell \\
\min \left\{r,\left\lfloor\frac{n-d+\ell}{2}\right\rfloor\right\}
\end{array}\right)+(d-\ell)\left(\begin{array}{c}
d \\
\min \{r-1,\lfloor d / 2\rfloor\}
\end{array}\right) .
$$

Note that $h_{\mathrm{Sp}}(n, \ell, r, d)=f(n, n+\ell, r, d)$. So Claim 67 implies (in the appendix) that for given positive $n, r$, and $\ell \geqslant 0$, the function $h_{\mathrm{Sp}}(n, \ell, r, d)$ is convex for $\ell \leqslant d \leqslant n$. 
Theorem 25. Let $n, d, r, \ell$ be integers with $0 \leqslant \ell<d \leqslant\left\lfloor\frac{n+\ell-1}{2}\right\rfloor$. If $G$ is an $n$-vertex graph with minimum degree $\delta(G) \geqslant d$, and $G$ is not $\ell$-hamiltonian, then

$$
N_{\mathrm{Sp}}(G, r) \leqslant \max \left\{h_{\mathrm{Sp}}(n, \ell, r, d), h_{\mathrm{Sp}}\left(n, \ell, r,\left\lfloor\frac{n+\ell-1}{2}\right\rfloor\right)\right\} .
$$

Proof. Let $\mathcal{C}$ be a Sperner family of cliques of size at most $r$ in $G$. Suppose that $N_{\mathrm{Sp}}\left(G, K_{r}\right)>h_{\mathrm{Sp}}(n, \ell, r,\lfloor(n+\ell-1) / 2\rfloor)$. By a generalization of Pósa's theorem (Lemma 8 in [9]), there exists some $\ell<k<\lfloor(n+\ell-1) / 2\rfloor$ such that $V(G)$ contains a subset $D$ of $k-\ell$ vertices with degree at most $k$ (and so $k \geqslant \delta(G) \geqslant d$ ).

For each vertex $v \in D, v$ is contained in at most $\left(\begin{array}{c}k \\ \min \{k / 2, r-1\}\end{array}\right)$ cliques of $\mathcal{C}$, and $G-D$ contains at most $\left(\begin{array}{c}n-k+\ell \\ \min \{\lfloor(n-k+\ell) / 2\rfloor, r\}\end{array}\right)$ cliques of $\mathcal{C}$. Hence $|\mathcal{C}| \leqslant N_{\mathrm{Sp}}(G, r) \leqslant h_{\mathrm{Sp}}(n, \ell, r, k) \leqslant$ $h_{\mathrm{Sp}}(n, \ell, r, d)$.

Our new result is:

Theorem 26. Let $n, k \geqslant 4$. Let $G$ be an n-vertex 2 -connected graph. If

$$
N_{\mathrm{Sp}}(G, r)>\frac{n-2}{k-3}\left(\begin{array}{c}
k-1 \\
\min \{r,\lfloor(k-1) / 2\rfloor\}
\end{array}\right),
$$

then $G$ is k-path connected.

Proof of Theorem 26. We use induction on $n$. If $n \leqslant k-1$, then by Theorem 19,

$$
N_{\mathrm{Sp}}(G, r) \leqslant\left(\begin{array}{c}
n \\
\min \{r,\lfloor n / 2\rfloor
\end{array}\right)=\frac{n-2}{k-3}\left(\frac{k-3}{n-2}\left(\begin{array}{c}
n \\
\min \{r,\lfloor n / 2\rfloor\}
\end{array}\right)\right) .
$$

And for $n \leqslant k-1$,

$$
\frac{k-3}{n-2}\left(\begin{array}{c}
n \\
\min \{r,\lfloor n / 2\rfloor\}
\end{array}\right) \leqslant \frac{k-3}{(k-1)-2}\left(\begin{array}{c}
k-1 \\
\min \{r,\lfloor(k-1) / 2\rfloor\}
\end{array}\right)=\left(\begin{array}{c}
k-1 \\
\min \{r,\lfloor(k-1) / 2\rfloor\}
\end{array}\right) .
$$

Hence (1) does not hold.

If $n=k$, consider any $x, y \in V(G)$ such that there is no hamiltonian $x, y$-path in $G$. If $x y \in E(G)$, then $G$ is not 1-hamiltonian, then by Theorem 25 with $d=2$ (since $G$ is 2-connected),

$$
\begin{aligned}
N_{\mathrm{Sp}}(G, r) & \leqslant \max \left\{h_{\mathrm{Sp}}(n, 1, r, 2), h_{\mathrm{Sp}}(n, 1, r,\lfloor n / 2\rfloor)\right) \\
& =h_{\mathrm{Sp}}(n, 1, r, 2) \\
& =\left(\begin{array}{c}
k-1 \\
\min \{r,\lfloor(k-1) / 2\rfloor\}
\end{array}\right)+2 \\
& \leqslant\left(\begin{array}{c}
k-1 \\
\min \{r,\lfloor(k-1) / 2\rfloor\}
\end{array}\right) \frac{k-2}{k-3}=\left(\begin{array}{c}
k-1 \\
\min \{r,\lfloor(k-1) / 2\rfloor\}
\end{array}\right) \frac{n-2}{k-3},
\end{aligned}
$$

and (1) again does not hold. If $x y \notin E(G)$, then the graph $G^{\prime}:=G \cup x y$ satisfies $N_{\mathrm{Sp}}\left(G^{\prime}, r\right) \geqslant N_{\mathrm{Sp}}(G, r)$, and $G^{\prime}$ is not 1-hamiltonian. So again we obtain $N_{\mathrm{Sp}}(G, r) \leqslant$ $N_{\mathrm{Sp}}\left(G^{\prime}, r\right) \leqslant\left(\begin{array}{c}k-1 \\ \min \{r,\lfloor(k-1) / 2\rfloor\}\end{array}\right) \frac{n-2}{k-3}$.

Thus from now on we may assume $n \geqslant k+1$. 
Claim 27. $G$ is 3-connected.

Proof. Suppose $\left\{v_{1}, v_{2}\right\}$ is a separating set. Let $C_{1}$ be the vertex set of a component of $G-\left\{v_{1}, v_{2}\right\}$ and $C_{2}=V(G)-\left\{v_{1}, v_{2}\right\}-C_{1}$. For $i=1,2$, let $G_{i}$ be obtained from $G-C_{3-i}$ by adding edge $v_{1} v_{2}$ if it is not in $G$. Let $n_{i}=\left|V\left(G_{i}\right)\right|$. By construction, each of $G_{1}$ and $G_{2}$ is 2-connected. Also,

$$
n_{1}+n_{2}=n+2 \quad \text { and } \quad N_{\mathrm{Sp}}(G, r) \leqslant N_{\mathrm{Sp}}\left(G_{1}, r\right)+N_{\mathrm{Sp}}(2, r) .
$$

By (2), some of $G_{i}$ satisfies (1). By symmetry, suppose $G_{2}$ does. If $x, y \in V\left(G_{2}\right)$, then we are done by induction. Suppose neither of $x$ and $y$ is in $V\left(G_{2}\right)$. Then by induction, $G_{2}$ has a $v_{1}, v_{2}$-path $P$ with at least $k$ vertices. Also, the 2-connected graph $G_{1}$ has two disjoint paths $P_{1}$ and $P_{2}$ from $\{x, y\}$ to $\left\{v_{1}, v_{2}\right\}$. Then $P_{1} \cup P \cup P_{2}$ forms a long $x, y$-path.

Finally, suppose $x \in V\left(G_{2}\right)$ and $y \notin V\left(G_{2}\right)$. Again by induction, $G_{2}$ has a $v_{1}, x$-path $P$ with at least $k$ vertices. Also, the 2 -connected graph $G_{1}$ has a $v_{1}, y$-path $P_{1}$ that avoids $v_{2}$. Then $P \cup P_{1}$ is what we need.

Claim 28. $\delta(G) \geqslant \frac{k+1}{2}$.

Proof. Suppose $v_{1} \in V(G)$ and $d\left(v_{1}\right) \leqslant k / 2$. Since $G$ is 3 -connected, we can choose a neighbor $v_{2}$ of $v_{1}$ so that $v_{2} \notin\{x, y\}$. Let $G^{\prime}$ be obtained from $G$ by contracting $v_{1}$ and $v_{2}$ into a new vertex that we again will call $v_{1}$. Since $G$ was 3 -connected, $G^{\prime}$ is 2 -connected.

Let $\mathcal{S}_{G}$ be a maximum Sperner family of cliques of size at most $r$ in $G$. We construct a family $\mathcal{S}^{\prime}$ of cliques of size at most $r$ in $G^{\prime}$ from $\mathcal{S}_{G}$ by

(a) deleting from $\mathcal{S}_{G}$ all cliques containing $v_{1}$; and

(b) replacing each clique $S \in \mathcal{S}_{G}$ with $v_{2} \in S$ and $v_{1} \notin S$ with the clique $S-v_{2}+v_{1}$.

We claim that $\mathcal{S}^{\prime}$ is Sperner. Indeed, suppose $S_{1}, S_{2} \in \mathcal{S}^{\prime}$ and $S_{1} \subset S_{2}$. Since $\mathcal{S}_{G}$ was Sperner, $v_{1} \in S_{2}-S_{1}$. But then $S_{2}-v_{1}+v_{2} \in \mathcal{S}_{G}$ and $S_{1} \subset S_{2}-v_{1}+v_{2}$.

By construction and Theorem 19,

$$
\left|\mathcal{S}_{G}\right|-\left|\mathcal{S}^{\prime}\right| \leqslant\left(\begin{array}{c}
d\left(v_{1}\right) \\
\min \left\{r-1,\left\lfloor d\left(v_{1}\right) / 2\right\rfloor\right\}
\end{array}\right) \leqslant\left(\begin{array}{c}
\lfloor k / 2\rfloor \\
\min \{r,\lfloor k / 4\rfloor\}
\end{array}\right) .
$$

But

$$
\left(\begin{array}{c}
\lfloor k / 2\rfloor \\
\min \{r,\lfloor k / 4\rfloor\}
\end{array}\right) \leqslant \frac{1}{k-3}\left(\begin{array}{c}
k-1 \\
\min \{r,\lfloor(k-1) / 2\rfloor\}
\end{array}\right)
$$

and hence $G^{\prime}$ satisfies (1). So by the minimality of $G$, graph $G^{\prime}$ has a long $x, y$-path. But then $G$ also does.

Applying Theorem 24 completes the proof of our theorem. 


\section{Constructing happy incidence bigraphs}

\subsection{Language of layered $r^{-}$-bigraphs}

A layered bigraph is a bigraph $G=(A, Y ; E)$ in which parts $A$ and $Y$ are ordered. An $r^{-}$-bigraph is a layered bigraph $G=(A, Y ; E)$ with $d(a) \leqslant r$ for each $a \in A$. Given a layered bigraph $G=(A, Y ; E)$, its shadow $\partial(G)$ is the graph $F$ with vertex set $Y$ such that $x y \in E(F)$ iff there is $a \in A$ with $\{x, y\} \subseteq N(a)$. A layered bigraph $G=(A, Y ; E)$ is Sperner if the family $\{N(a): a \in A\}$ is Sperner. By definition, if $N(a)=\{v, u\}$ in a Sperner bigraph, then the codegree of the pair $v u$ is 1 . In particular, the incidence graph $G_{\mathcal{H}}$ of an $r^{-}$-graph $\mathcal{H}$ is a Sperner $r^{-}$-bigraph if and only if $\mathcal{H}$ is Sperner.

A vertex $a \in A$ of a layered bigraph $G=(A, Y ; E)$ is happy, if the the codegree $d(x, y)$ of each pair $\{x, y\} \subseteq N(a)$ is at least $d(a)-1$ (and unhappy otherwise). A layered bigraph $G=(A, Y ; E)$ is happy if every vertex $a \in A$ is happy.

Below we will show a useful property of happy graphs. We first prove a simple corollary of Hall's Theorem.

Lemma 29 (Folklore). Let $G=(A, B ; E)$ be a bipartite graph with no isolated vertices such that for each $a \in A$ and every $b \in N(A), d(a) \geqslant d(b)$. Then $G$ has a matching covering $A$.

Proof. Suppose that $G$ has no matching covering $A$. By Hall's Theorem, there is $S \subseteq A$ with $|S|>|N(S)|$. Choose a minimum such $S$, say $S=\left\{a_{1}, \ldots, a_{s}\right\}$. By the minimality of $S, G$ has a matching $M$ covering $S^{\prime}:=S-a_{s}$, say $M=\left\{a_{i} b_{i}: 1 \leqslant i \leqslant s-1\right\}$. Since $|N(S)| \leqslant s-1$, we have $N(S)=\left\{b_{1}, \ldots, b_{s-1}\right\}$. So,

$d\left(a_{1}\right)+\ldots+d\left(a_{s-1}\right)+d\left(a_{s}\right)=e(S, N(S))=d_{S}\left(b_{1}\right)+\ldots+d_{S}\left(b_{s-1}\right) \leqslant d\left(a_{1}\right)+\ldots d\left(a_{s-1}\right)$,

a contradiction.

Lemma 30. Let $r \geqslant 3$. If $G=(A, Y ; E)$ is a happy Sperner $r^{-}$-bigraph and $\partial(G)$ contains a cycle of length $\ell \geqslant r$, then $G$ contains a cycle of length $2 \ell$.

Proof. Let $C=x_{1}, \ldots, x_{\ell}$ be a cycle of length $\ell \geqslant r$ in $\partial(G)$. Let $F$ be the bipartite graph with parts $Q=E(C)$ and $A$ such that a pair $\left(x_{i} x_{i+1}, a\right)$ is an edge in $F$ if and only if $\left\{x_{i} x_{i+1}\right\} \subseteq N(a)$. If $\ell \geqslant r+1$, then since each $a \in A$ has degree less than $\ell, a$ is adjacent to at most $d(a)-1$ pairs $x_{i} x_{i+1}$. On the other hand, for each edge $\left(x_{i} x_{i+1}, a\right)$ in $F, d_{F}\left(\left\{x_{i} x_{i+1}\right\}\right) \geqslant d(a)-1$ since $G$ is happy. So by the previous lemma, $F$ has a matching that covers $E(C)$, say with $x_{i} x_{i+1}$ matched to $f\left(x_{i} x_{i+1}\right) \in A$. Then we obtain the cycle $x_{1}, f\left(x_{1} x_{2}\right), x_{2}, f\left(x_{2} x_{3}\right), \ldots, x_{\ell}, f\left(x_{\ell} x_{1}\right), x_{1}$ of length $2 \ell$ in $G$.

Now suppose $\ell=r$. If for every $a \in A, N_{G}(a) \neq\left\{x_{1}, \ldots, x_{r}\right\}$, then $d_{F}(a) \leqslant d(a)-1$, and we are done as in the previous case. So suppose there exists an $a$ such that $N_{G}(a)=$ $\left\{x_{1}, \ldots, x_{r}\right\}$. Then because $G$ is Sperner, each $a^{\prime} \in A-a$ is adjacent to at most $r-1$ vertices in $\left\{x_{1}, \ldots, x_{r}\right\}$, and hence $d_{F}\left(a^{\prime}\right) \leqslant(r-1)-1$. Consider the graph $F-a$. For $a^{\prime} \in A-a$,

$$
d_{F-a}\left(a^{\prime}\right)=d_{F}\left(a^{\prime}\right) \leqslant \min \left\{r-2, d\left(a^{\prime}\right)-1\right\} .
$$


If some vertex $x_{i} x_{i+1}$ was adjacent to $a$ in $F$, then $d_{F}\left(x_{i} x_{i+1}\right) \geqslant d(a)-1=r-1$ and so $d_{F-a}\left(x_{i} x_{i+1}\right) \geqslant r-2$. Otherwise, for each $x_{i} x_{i+1}$ not adjacent to $a$ in $F$, and each $a^{\prime} \in N_{F}\left(x_{i}, x_{i+1}\right), d_{F-a}\left(x_{i} x_{i+1}\right)=d_{F}\left(x_{i} x_{i+1}\right) \geqslant d\left(a^{\prime}\right)-1$, so we are finished as in the first case.

The same proof also yields the following Lemma for paths of any length.

Lemma 31. Let $G=(A, Y ; E)$ be a happy $r^{-}$-bigraph. If $\partial(G)$ contains a path with $\ell$ vertices, then $G$ contains a path with $2 \ell-1$ vertices with endpoints in $Y$.

A vertex $y \in Y$ of degree 2 in is special, if each of the two neighbors is either unhappy or also has degree 2 .

Vertices $x, y \in Y$ and $a \in A$ form a special triple if $x$ and $y$ are special (in particular they have degree 2), $N(a)=\{x, y\}$, and the other neighbors of $x$ and $y$ are unhappy.

For each graph $H$, the circumference, $c(H)$, is the length of a longest cycle in $H$.

We will often use the following known property of 2-connected graphs.

Lemma 32. Let $G$ be a 2-connected graph, $x y \in E(G)$ and $S \subset V(G)$ with $|S| \leqslant$ $|V(G)|-2$.

(1) $G-x y$ is 2-connected iff $G-x y$ has a cycle containing $x$ and $y$;

(2) the graph $G / S$ obtained by gluing the vertices of $S$ into one vertex $s^{*}$ is 2 -connected iff $s^{*}$ is not a cut vertex of $G / S$.

\subsection{Unhappy $\boldsymbol{r}^{-}$-bigraphs}

Definition 33. Let $G=(A, Y ; E)$ be a Sperner layered 2-connected $r^{-}$-bigraph $G=$ $(A, Y ; E)$. A shrinking of $\boldsymbol{G}$ is one of the following operations:

1. deleting an edge of $G$ incident to an unhappy vertex,

2. deleting a special vertex $y \in Y$ and all neighbors $b \in N(y)$ with $d(b)=2$,

3. deleting a special triple $x, y \in Y$ and $a \in A$, or

4. gluing together all but one of the neighbors of some unhappy vertex $a \in A$.

We will say that an unhappy Sperner layered 2-connected $r^{-}$-bigraph $G$ with $c(G)<2 k$ admits a shrinking if after a shrinking of $G$ the resulting graph $G^{\prime}$ satisfies all the properties (S1)-(S5) below:

(S1) $G^{\prime}$ is 2-connected;

(S2) $\left|E^{\prime}\right| \leqslant|E|,\left|Y^{\prime}\right| \leqslant|Y|$, and $\left|E^{\prime}\right|+\left|Y^{\prime}\right|<|E|+|Y|$;

(S3) $G^{\prime}$ is Sperner; 
(S4) $|A|-\left|A^{\prime}\right| \leqslant|Y|-\left|Y^{\prime}\right| ;$ and

(S5) $c\left(G^{\prime}\right)<2 k$.

The goal of this subsection is to prove that each unhappy Sperner layered 2-connected $r^{-}$-bigraph either admits a shrinking or has a special structure and high maximum average degree. The main result of the subsection is the following lemma.

Lemma 34. Suppose $k \geqslant r \geqslant 3$ are integers. Let $G=(A, Y ; E)$ be a Sperner layered 2connected $r^{-}$-bigraph with $c(G)<2 k$. If $G$ is not happy, then either $G$ admits a shrinking or for every unhappy vertex $a \in A$, there exist three vertices $y_{1}, y_{2}, y_{3} \in N(a)$ and three subgraphs $B_{1}, B_{2}, B_{3}$ of $G$ such that for $i \in\{1,2,3\}$ :

(B1) $y_{i} \in V\left(B_{i}\right), a \notin V\left(B_{i}\right)$, and $y_{i}$ is the only neighbor of a in $B_{i}$;

(B2) $B_{i}$ is 2-connected and Sperner;

(B3) there exists a $x_{i} \in Y$ such that $\left\{a, x_{i}\right\}$ separates $B_{i}$ from $G-B_{i}$;

(B4) $G-\left(B_{i}-x_{i}\right)-a$ is Sperner and 2-connected; and

(B5) for $j \in\{1,2,3\}-\{i\},\left|V\left(B_{i}\right) \cap V\left(B_{j}\right)\right| \leqslant 1$ with equality if and only if $x_{i}=x_{j}$.

Proof. Suppose, $G=(A, Y ; E)$ is a Sperner layered 2-connected $r^{-}$-bigraph with $c(G)<$ $2 k$ that is not happy. Then it has an unhappy vertex $a \in A$. Let $N_{G}(a)=\left\{y_{1}, \ldots, y_{t}\right\}$. Since $a$ is unhappy, $t \geqslant 3$. We derive a series of properties of such $G$.

A vertex $y_{i} \in N(a)$ is an a-menace, if there is a vertex $m\left(a, y_{i}\right) \in A-a$ such that $N(a)-y_{i} \subseteq N\left(m\left(a, y_{i}\right)\right)$. Since $G$ is Sperner,

$$
G-a y_{i} \text { is Sperner if and only if } y_{i} \text { is not an a-menace. }
$$

For brevity, we call pairs of vertices in $Y$ of codegree 1 thin and of codegree at least 2 - thick.

Claim 35. If every pair in $N(a)$ is thick, then for some $i$, the graph $G$-ay satisfies (S1)-(S5). In particular, $G$ admits a shrinking of type (1).

Proof. For each $y_{i} \in N(a)$, the graph $G_{i}:=G-a y_{i}$ trivially satisfies (S2), (S4), and (S5) in the definition of shrinking. We will show that every $G_{i}$ is also 2-connected, i.e., it satisfies (S1). Let $y_{j}, y_{k} \in N(a)-y_{i}$. Because every pair of $N(a)$ is thick, there exists distinct vertices $b_{i j}, b_{i k} \neq a$ such that $\left\{y_{i}, y_{j}\right\} \in N\left(b_{i j}\right)$ and $\left\{y_{i}, y_{k}\right\} \in N\left(b_{i k}\right)$. Applying Lemma 32 with the cycle $y_{i} b_{i j} y_{j} a y_{k} b_{i k} y_{i}$ certifies that $G_{i}$ is 2-connected.

If for some $1 \leqslant i \leqslant t$, the graph $G_{i}$ is Sperner, i.e., satisfies (S3), then we are done. Assume not. Because $a$ is the only vertex with a changed neighborhood in $G_{i}$, for all $i$ there exists a vertex $b_{i}$ in $G$ such that $\left\{y_{1}, \ldots, y_{t}\right\}-\left\{y_{i}\right\} \subset N\left(b_{i}\right)$. Furthermore, for $i \neq j$, $b_{i} \neq b_{j}$, otherwise some $N\left(b_{i}\right)$ contains $N(a)$, contradicting the fact that $G$ is Sperner.

In particular, each pair in $N(a)$ belongs in the neighborhoods of $a$ and $d(a)-2$ additional vertices, contradicting that $a$ is unhappy. 
Claim 36. If $G$ does not admit a shrinking of type (1), then no two thick pairs in $N(a)$ share a vertex.

Proof. Suppose none of the graphs $G-a y_{i}$ satisfies (S1)-(S5). By Claim 35, $N(a)$ contains a thin pair. First we show that there exist some thick pairs $\left\{y_{i^{*}}, y_{j^{*}}\right\},\left\{y_{i^{*}}, y_{k^{*}}\right\}$ and a thin pair $\left\{y_{s^{*}}, y_{t^{*}}\right\}$ such that $s^{*}, t^{*} \neq i^{*}$. Let $\left\{y_{i}, y_{j}\right\}$ and $\left\{y_{i}, y_{k}\right\}$ be some intersecting thick pairs and $\left\{y_{s}, y_{t}\right\}$ be a thin pair in $N(a)$, where without loss of generality, $y_{s} \notin\left\{y_{i}, y_{j}\right\}$. If $y_{t} \neq y_{i}$ then we are done. If not then consider instead the pair $\left\{y_{s}, y_{j}\right\}$. If it is thin, then we take this pair instead of $\left\{y_{s}, y_{t}\right\}$. If it is thick, then we let $\left\{y_{i}, y_{j}\right\},\left\{y_{s}, y_{j}\right\}$ be our intersecting thick pairs with $y_{j}$ playing the role of $y_{i^{*}}$ and $\left\{y_{s}, y_{t}\right\}=\left\{y_{s}, y_{i}\right\}$ be the thin pair.

Now consider the graph $G-a y_{i^{*}}$. As in the previous claim, it satisfies (S2), (S4), and (S5) as well as (S1) in the definition of shrinking where we define vertices $b_{i^{*} j^{*}}, b_{i^{*} k^{*}}$ similarly. Since no other vertex contains the pair $\left\{y_{s^{*}}, y_{t^{*}}\right\}$ in its neighborhood, $G-a y_{i^{*}}$ is Sperner, a contradiction.

Claim 37. If $G$ does not admit a shrinking of type (1), then the codegree of each pair in $N(a)$ is at most 2 .

Proof. Suppose $G$ does not admit a shrinking of type (1), but there exist distinct vertices $b_{1}, b_{2} \neq a$ both adjacent to $y_{1}$ and $y_{2}$. Since $\left\{y_{1}, y_{2}\right\}$ is a thick pair, by Claim 36, $\left\{y_{1}, y_{3}\right\}$ and $\left\{y_{2}, y_{3}\right\}$ are thin. Let $P$ be a shortest path in $G-a$ from $y_{3}$ to $\left\{y_{1}, y_{2}\right\}$. Note that if $P$ contains $b_{1}$ or $b_{2}$, then by the minimality of $|P|$, either $y_{1}$ or $y_{2}$ follows directly after. Therefore we may assume by symmetry that $y_{1} \in P$ and $b_{2} \notin P$. Consider the graph $G-a y_{1}$. Trivially it satisfies (S2), (S4), and (S5). Because $\left\{y_{2}, y_{3}\right\}$ is thin, it also satisfies (S3). Finally, the cycle $y_{3} P y_{1} b_{2} y_{2} a y_{3}$ certifies that (S1) is satisfied.

Claim 38. If $G$ does not admit a shrinking of type (1) and a proper subset $S$ of $N(a)$ is a separating set in $G$, then $S$ contains an a-menace.

Proof. Suppose $G$ does not admit a shrinking of type (1) but the claim does not hold. Choose a smallest separating subset $S=\left\{y_{1}, \ldots, y_{s}\right\}$ of $N(a)$ not containing $a$-menaces. Since $S$ is a proper subset of $N(a), s<t$. Let $D_{1}$ and $D_{2}$ be components of $G-S$, where $D_{1}$ contains $a$. By the minimality of $S$,

$$
\text { each } y_{i} \in S \text { has a neighbor in } D_{2} \text {. }
$$

Since $G$ is 2-connected, there are two $y_{t}, S$-paths $P_{1}$ and $P_{2}$ sharing only $y_{t}$. By symmetry we may assume that $P_{1}$ avoids $a$. Let $y_{1}$ be the end of $P_{1}$ in $S$. By (4), there is a $y_{1}, y_{2}$-path $P_{3}$ all whose internal vertices are in $D_{2}$.

Consider $G-a y_{1}$. Properties (S2), (S4) and (S5) hold by definition. Since $y_{1}$ is not an $a$-menace, by (3), $G-a y_{1}$ is Sperner, i.e. (S3) holds. Cycle $y_{2} a y_{t} P_{1} y_{1} P_{3} y_{2}$ together with Lemma 32 show that $G-a y_{1}$ is 2 -connected. Thus, $G-a y_{1}$ satisfies the lemma.

Claim 39. Suppose $G$ admits no shrinkings of types (1) and (2). Then $N($ a) has no thick pairs. 
Proof. Suppose pair $y_{1} y_{2}$ is thick. By Claims 36 and $37, d\left(y_{1} y_{2}\right)=2$, and the common neighbor $b \in A-a$ of $y_{1}$ and $y_{2}$ has no other neighbors in $N(a)$. Let $N(b)=$ $\left\{y_{1}, y_{2}, z_{1}, \ldots, z_{s}\right\}$. Since $G$ is Sperner, $s \geqslant 1$.

By Claim 36, neither of $y_{1}$ and $y_{2}$ is an $a$-menace. So, by Claim 38, $G-y_{1}-y_{2}$ contains an $a, b$-path $P_{1}$. We may assume that $y_{t}$ is the second and $z_{1}$ is the second to last vertices of $P_{1}$. Since $d\left(y_{1} y_{2}\right)=2$, by Claim 36, $z_{1} \notin N(a)$. So $y_{t} \neq y_{1}$.

Case 1: $d\left(y_{1}\right)=2$. Then $d\left(y_{1} z_{1}\right)=1$ and hence $b$ is unhappy. So, since $d\left(y_{1} y_{2}\right)=2$, by Claim 36, $d\left(y_{2} z_{1}\right)=1$. Consider $G^{\prime}=G-y_{1}$. As in the proof of Claim 38, (S2), (S4) and (S5) hold for $G^{\prime}$ by definition. Cycle $y_{2} a y_{2} P_{1} b y_{2}$ together with Lemma 32 certify that $G^{\prime}$ is 2-connected, i.e., (S1) holds. Only the neighborhoods of $a$ and $b$ in $A^{\prime}$ are distinct from those in $A$. So the fact that $d\left(y_{2} z_{1}\right)=d\left(y_{2} y_{t}\right)=1$ shows that $G^{\prime}$ is Sperner. Thus, deleting $y_{1}$ is a shrinking of type 2 . This proves Case 1 .

Case 2: $d\left(y_{1}\right) \geqslant 3$. Let $c \in N\left(y_{1}\right)-a-b$, where if possible we choose $c$ to be adjacent to $z_{1}$. Since $G$ is 2 -connected, $G-y_{1}$ has a shortest path $P_{2}$ from $c$ to $V\left(P_{1}\right) \cup\left\{y_{2}\right\}$. Let $x$ be the end of $P_{2}$ in $V\left(P_{1}\right) \cup\left\{y_{2}\right\}$.

Case 2.1: $x \neq b$. Consider $G^{\prime}=G-a y_{1}$. As above, (S2), (S4) and (S5) trivially hold for $G^{\prime}$. Since only the neighborhood of $a$ in $A^{\prime}$ is distinct from those in $A$ and $d\left(y_{2} y_{t}\right)=1, G^{\prime}$ is Sperner. We need now only to show that $G^{\prime}$ is 2-connected. If $x=y_{2}$, then cycle $c P_{2} y_{2} a P_{1} b y_{1} c$ certifies this. If $x \in V\left(P_{1}\right)-b$, then our certificate is cycle $c y_{1} b y_{2} a P_{1}(a, x) x P_{2} c$, where $P_{1}(a, x)$ denotes the subpath of $P_{1}$ from $a$ to $x$.

Case 2.2: $x=b$. Note that because $x \neq z_{1}$, by the choice of $c$ and the choice of $P_{2}$, $z_{1} \notin N(c)$ for any $c \in N\left(y_{1}\right)-a-b$. In particular, $d\left(y_{1} z_{1}\right)=1$, and so $b$ is unhappy. The second to last vertex of $P_{2}$ is none of $z_{1}, y_{1}, y_{2}$, so we may assume it is $z_{2}$. Consider $G^{\prime}=G-b y_{1}$. Cycle $c P_{2} b y_{2} a y_{1} c$ shows that $G^{\prime}$ is 2 -connected. As above, (S2), (S4) and (S5) trivially hold for $G^{\prime}$. Thus if $G^{\prime}$ is Sperner, then the claim is proved. If $G^{\prime}$ is not Sperner, then $y_{1}$ is a $b$-menace, and there is a vertex $g \in A-b$ such that $N(g) \supset\left\{y_{2}, z_{1}, z_{2}\right\}$. Since $z_{1} a \notin E, g \neq a$. But then instead of the path $P_{2}$, we can consider the path $P_{2}\left(c, z_{2}\right) z_{2} g z_{1}$, and will have Case 2.1.

Claim 40. Suppose all pairs in $N(a)$ are thin. Let $G_{t}$ be obtained from $G$ by gluing all vertices in $N(a)-y_{t}$ into one vertex $y^{*}$. If $G^{\prime}$ is Sperner, then $G_{t}$ satisfies (S1)-(S5). In particular, $G$ admits a shrinking of type (4).

Proof. (S2) holds for $G_{t}$ trivially. When gluing the vertices, we lose edges only if some pair $y_{i}, y_{j} \in N(a)$ have a common neighbor. But because $\left\{y_{i}, y_{j}\right\}$ is thin, they have no common neighbors other than $a$. Hence $\left|E^{\prime}\right|=|E|-(t-2)$ and $\left|Y^{\prime}\right|=|Y|-(t-2)$ so (S4) holds. Property (S5) is less clear but still is true: If $G_{t}$ has a cycle $C$ of length at least $2 k$, then it must go through $y^{*}$. Furthermore, if $C$ does not go through $a$, then either $C$ is present in $G$ with $y^{*}$ replaced by some $y_{i}$, or it can be extended through $a$ connecting some $y_{i}$ and $y_{j}$. If $C$ does through $a$, then it uses edges $a y_{t}$ and $a y^{*}$; we can modify $C$ in $G$ to a cycle of the same length. Thus, (S5) also holds.

Since all pairs in $N(a)$ are thin, none of $y_{i}$ is an $a$-menace. So by Claim 38 and Lemma $32, G_{t}$ is 2-connected hence satisfies (S1). 
Assume $G_{t}$ is not Sperner but all pairs in $N(a)$ are thin. Then $N_{G_{t}}(a)$ is not contained in any other neighborhood. By symmetry there are $b_{1}, b_{2} \in A-a$ such that $N_{G}\left(b_{2}\right)-y_{2} \subset$ $N_{G}\left(b_{1}\right)$ and $y_{1} b_{1} \in E$. Note that each of $b_{1}$ and $b_{2}$ has exactly one neighbor in $N(a)\left(y_{1}\right.$ and $y_{2}$ respectively), and there is $x \in N\left(b_{1}\right) \cap N\left(b_{2}\right)$ such that $x \notin N(a)$.

Claim 41. If $d\left(b_{2}\right) \geqslant 3$, then $G-$ ay $y_{2}$ satisfies (S1)-(S5). In particular, $G$ admits a shrinking of type (1).

Proof. Suppose $N\left(b_{2}\right) \supseteq\left\{y_{2}, x_{1}, x_{2}\right\}$. Then by the definition of $b_{1}, N\left(b_{1}\right) \supseteq\left\{y_{1}, x_{1}, x_{2}\right\}$. So by Claim 39 applied to $b_{1}$ and $b_{2}$, because the pair $\left\{x_{1}, x_{2}\right\}$ is thick, both $b_{1}$ and $b_{2}$ are happy. Since $G$ is 2-connected, $G-a$ has a shortest path $P$ from $y_{t}$ to $Z=$ $\left\{y_{1}, y_{2}, b_{1}, b_{2}, x_{1}, x_{2}\right\}$. Let $z$ be the last vertex of $P$. By symmetry, we may assume $z \in$ $\left\{y_{2}, b_{2}, x_{2}\right\}$. Consider $G-a y_{2}$. As before, (S2),(S4) and (S5) hold. Since all pairs in $N(a)$ are thin, $G-a y_{2}$ is Sperner. If $z=y_{2}$, then the cycle $a y_{t} P y_{2} b_{2} x_{2} b_{1} y_{1} a$ shows that $G-a y_{2}$ is 2 -connected.

So suppose $z \in\left\{b_{2}, x_{2}\right\}$. Since $b_{2}$ is happy, there is another $b_{3}$ adjacent to $y_{2}$ and $x_{2}$. By definition, it is distinct from $b_{1}$ and $a$. So if $z=x_{2}$ and $P$ does not pass through $b_{3}$, then we have cycle $a y_{t} P x_{2} b_{3} y_{2} b_{2} x_{1} b_{1} y_{1} a$. Similarly, if $z=b_{2}$ and $P$ does not pass through $b_{3}$, then we have cycle $a y_{t} P b_{2} y_{2} b_{3} x_{1} b_{1} y_{1} a$. Finally, if $P$ passes through $b_{3}$, then we have cycle $a y_{t} P\left(a, b_{3}\right) b_{3} y_{2} b_{2} x_{1} b_{1} y_{1} a$.

So assume $d\left(b_{2}\right)=2$. Recall $x=N\left(b_{1}\right) \cap N\left(b_{2}\right)$.

Claim 42. Suppose $d\left(y_{2}\right)=2$. Then either $G-b_{2}-y_{2}$ or $G-a y_{1}$ satisfies (S1)-(S5), $b_{1}$ is unhappy and $G-b_{1} x$ satisfies $(\mathrm{S} 1)-(\mathrm{S} 5)$, or $d(x)=2$, both neighbors of $x$ are unhappy, and $G-b_{2}-y_{2}-x$ satisfy (S1)-(S5). In particular, $G$ admits a shrinking either of type (1), type (2), or type (3).

Proof. Assume $N\left(y_{2}\right)=\left\{a, b_{2}\right\}$. By Claim 38, $G-y_{1}-y_{2}$ has an $a, x$-path $P$. We can choose a shortest such path. Let $c$ be the second to last vertex in $P$.

Case 1: $c \neq b_{1}$. Consider $G^{\prime}=G-b_{2}-y_{2}$. As before, (S2),(S4) and (S5) hold for $G^{\prime}$. Since all pairs in $N(a)$ are thin, $G^{\prime}$ is Sperner. The cycle $a P x b_{1} y_{1} a$ shows that $G^{\prime}$ is 2-connected.

Case 2: $c=b_{1}$. Let $z$ be the previous to $c$ vertex of $P$. Since all pairs in $N(a)$ are thin, $z \neq y_{t}$. If $b_{1}$ is happy, then there exists a vertex $b_{3} \neq b_{1}$ with $\left\{y_{1}, x\right\} \subseteq N\left(b_{3}\right)$. Then $b_{3}$ can play the role of $b_{1}$ in the definition of $b_{1}$ and $b_{2}$. In this case, we get Case 1 and are done. Thus, $b_{1}$ is unhappy. Hence all pairs in $N\left(b_{1}\right)$ are thin.

If $d(x)=2$, consider $G^{\prime}=G-b_{2}-y_{2}-x$. As before, (S2),(S4) and (S5) hold for $G^{\prime}$. Since all pairs in $N(a)$ and in $N\left(b_{1}\right)$ are thin, $G^{\prime}$ is Sperner. The cycle $a P\left(a, b_{1}\right) b_{1} y_{1} a$ shows that $G^{\prime}$ is 2-connected.

So suppose $b_{4} \in N(x)-b_{1}-b_{2}$. Since $G$ is 2-connected, $G-x$ has an $a, b_{4}$-path $P_{1}$. If $P_{1}$ does not intersect $\left\{b_{1}, y_{1}\right\}$, then we have Case 1 with $P=a P_{1} b_{4} x$. So, suppose $u$ is the first vertex in $\left\{b_{1}, y_{1}\right\}$ that is hit by $P_{1}$. Note that if $P_{1}$ meets $P-u$ before $u$, then we can modify it to avoid intersecting with $\left\{b_{1}, y_{1}\right\}$. Thus we assume below that this is not the case. 
If $u=y_{1}$, consider $G^{\prime}=G-a y_{1}$. As before, (S2),(S4) and (S5) hold for $G^{\prime}$. Since all pairs in $N(a)$ are thin, $G^{\prime}$ is Sperner. The cycle $a P\left(a, b_{1}\right) b_{1} y_{1} P_{1}\left(y_{1}, b_{4}\right) x b_{2} y_{2} a$ shows that $G^{\prime}$ is 2-connected. Finally, if $u=b_{1}$, consider $G^{\prime}=G-b_{1} x$. As before, (S2),(S4) and (S5) hold for $G^{\prime}$. Since all pairs in $N\left(b_{1}\right)$ are thin, $G^{\prime}$ is Sperner. The cycle $a P\left(a, b_{1}\right) b_{1} P_{1}\left(b_{1}, b_{4}\right) x b_{2} y_{2} a$ shows that $G^{\prime}$ is 2 -connected.

We now assume $d\left(y_{2}\right) \geqslant 3$.

Claim 43. Suppose the set $\left\{x, y_{1}, y_{2}\right\}$ does not separate a from $b_{1}$. Then either $G-a y_{1}$ satisfies (S1)-(S5), $b_{1}$ is unhappy and $G-b_{1} y_{1}$ satisfies (S1)-(S5), or $d\left(y_{1}\right)=2$, both neighbors of $y_{1}$ are unhappy, and $G-y_{1}$ satisfies (S1)-(S5). In particular, $G$ admits a shrinking either of type (1) or type (2).

Proof. We may find in $G-\left\{x, y_{1}, y_{2}\right\}$ an $a, b_{1}$-path $P$. Note that $b_{2} \notin P$ since $N\left(b_{2}\right)=$ $\left\{x, y_{2}\right\}$. Let the second vertex of $P$ be $y_{t}$.

If $b_{1}$ is happy, then there is $b_{3} \in A-b_{1}$ with $N\left(b_{3}\right) \supseteq\left\{y_{1}, x\right\}$. Consider $G^{\prime}=G-a y_{1}$. As before, (S2),(S4) and (S5) hold for $G^{\prime}$. Since all pairs in $N(a)$ are thin, $G^{\prime}$ is Sperner. We need to show that $G^{\prime}$ is 2-connected. If $b_{3} \in P$, then the cycle $a P\left(a, b_{3}\right) b_{3} y_{1} b_{1} x b_{2} y_{2} a$ certifies this. Otherwise, the cycle $a P b_{1} y_{1} b_{3} x b_{2} y_{2} a$ certifies this.

So, $b_{1}$ is unhappy, and all pairs in $N\left(b_{1}\right)$ are thin. If $d\left(y_{1}\right)=2$, consider $G^{\prime}=G-y_{1}$. As before, (S2),(S4) and (S5) hold for $G^{\prime}$. Since all pairs in $N\left(b_{1}\right)$ and in $N(a)$ are thin, $G^{\prime}$ is Sperner. The cycle $a P b_{1} x b_{2} y_{2} a$ shows that $G^{\prime}$ is 2-connected.

Thus, $d\left(y_{1}\right) \geqslant 3$. Let $c \in N\left(y_{1}\right)-a-b_{1}$. Let $P_{1}$ be a shortest path in $G-y_{1}$ from $c$ to $V(P) \cup\left\{x, y_{2}\right\}$. Let $z$ be the last vertex of $P_{1}$. If $z \in V(P)-b_{1}$, consider $G^{\prime}=G-a y_{1}$. As before, (S2),(S4) and (S5) hold for $G^{\prime}$. Since all pairs in $N(a)$ are thin, $G^{\prime}$ is Sperner. The cycle $a P(a, z) z P_{1} c y_{1} b_{1} x b_{2} y_{2} a$ certifies that $G^{\prime}$ is 2-connected.

If $z \in\left\{b_{1}, x, y_{2}\right\}$, consider $G^{\prime}=G-b_{1} y_{1}$. As before, (S2),(S4) and (S5) hold for $G^{\prime}$. Since all pairs in $N\left(b_{1}\right)$ are thin, $G^{\prime}$ is Sperner. Let $P_{2}$ denote the path $a y_{2} b_{2} x b_{1}$. Then the cycle $a y_{1} c P_{1} z P_{2}\left(z, b_{1}\right) b_{1} P a$ certifies that $G^{\prime}$ is 2 -connected.

Therefore we assume $\left\{x, y_{1}, y_{2}\right\}$ separates $a$ and $b_{1}$.

Claim 44. Suppose $\{x, a\}$ does not separate $y_{2}$ from $N(a)-y_{2}$. Then $G-a y_{2}$ satisfies (S1)-(S5). In particular, $G$ admits a shrinking of type (1).

Proof. Let $P$ be a shortest $a, x$-path in $G-y_{1}-y_{2}$. By Claim 43, $P$ does not go through $b_{1}$. Let the second vertex of $P$ be $y_{t}$. Let $P_{1}$ be a shortest path in $G-a-x$ from $y_{2}$ to $\left(N(a)-y_{2}\right) \cup V(P)$. Let $z$ be the last vertex of $P_{1}$. If $b_{1} \in V(P)$, then we can take $z=y_{1}$. Consider $G^{\prime}=G-a y_{2}$. As before, (S2),(S4) and (S5) hold for $G^{\prime}$. Since all pairs in $N(a)$ are thin, $G^{\prime}$ is Sperner. If $z \in N(a)-y_{t}$ then the cycle $y_{2} P_{1} z a P x b_{2} y_{2}$ certifies that $G^{\prime}$ is 2-connected. Otherwise, the cycle $y_{2} P_{1} z P(z, a) a y_{1} b_{1} x b_{2} y_{2}$ does it.

So suppose $\{x, a\}$ separates $y_{2}$ from the rest of $N(a)$. Let $C_{2}$ be the vertex set of the component of $G-a-x$ containing $y_{2}$ and let $G_{2}=G\left[C_{2} \cup\{a, x\}\right]$. By Claim 44, $C_{2} \cap N(a)=\left\{y_{2}\right\}$. If $x$ has no neighbors in $C_{2}-b_{2}$, then by Claim $44, y_{2}$ would be a 
cut vertex, a contradiction. Thus, in view of $b_{2}$, no vertex in $G_{2}-a$ separates $x$ from $y_{2}$. Since no vertex in $G_{2}-a$ may separate $\left\{y_{2}, x\right\}$ from any other vertex, we conclude

$$
G_{2}-a \text { is 2-connected and the unique neighbor of a in } C_{2} \text { is } y_{2} \text {. }
$$

Claim 45. Suppose $\{x, a\}$ does not separate $y_{1}$ from $N(a)-y_{1}$. Then one of the following occurs: $G-a y_{1}$ satisfies (S1)-(S5), $b_{1}$ is unhappy and for some $y \in N\left(b_{1}\right) G-b_{1} y$ satisfies (S1)-(S5), or $d\left(y_{1}\right)=2$, both neighbors of $y_{1}$ are unhappy, and $G-y_{1}$ satisfies (S1)-(S5). In particular, $G$ admits a shrinking either of type (1) or type(2).

Proof. If $d\left(b_{1}\right)=2$, then by symmetry of $b_{1}$ and $b_{2}$ and the previous claim, we are done. So $d\left(b_{1}\right) \geqslant 3$. Let $x^{\prime} \in N\left(b_{1}\right)-y_{1}-x$. Let $P$ be a shortest $a, x$-path in $G-y_{1}-y_{2}$. By Claim 44, $P$ does not go through $b_{2}$. Let the second vertex of $P$ be $y_{t}$.

Let $P_{1}$ be a minimal path in $G-a-x$ from $\left\{y_{1}, b_{1}\right\}$ to $V(P) \cup\left(N(a)-y_{1}-y_{2}\right)$. Let $z_{1}$ be the first vertex of $P_{1}$ and $z_{2}$ - the last. If $z_{1}=y_{1}$, consider $G^{\prime}=G-a y_{1}$. As above, (S2),(S4) and (S5) hold for $G^{\prime}$. Since all pairs in $N(a)$ are thin, $G^{\prime}$ is Sperner. If $z_{2} \in N(a)-y_{t}$ then the cycle $y_{1} P_{1} z_{2} a y_{2} b_{2} x b_{1} y_{1}$ certifies that $G^{\prime}$ is 2-connected. Otherwise, the cycle $y_{1} P_{1} z_{2} P\left(z_{2}, a\right) a y_{2} b_{2} x b_{1} y_{1}$ does it.

So suppose $z_{1}=b_{1}$.

Case 1: $b_{1}$ is unhappy. If $z_{2} \in V(P)$, then we consider $G^{\prime}=G-x b_{1}$. As above, (S2),(S4) and (S5) hold for $G^{\prime}$. Since $b_{1}$ is unhappy, all pairs in $N\left(b_{1}\right)$ are thin, and hence $G^{\prime}$ is Sperner. The cycle $b_{1} P_{1} z_{2} P\left(z_{2}, x\right) x b_{2} y_{2} a y_{1} b_{1}$ certifies that $G^{\prime}$ is 2 -connected. So below we assume $z_{2}=y_{3}$ and $t \geqslant 4$.

If $d\left(y_{1}\right)=2$, then we consider $G^{\prime}=G-y_{1}$. As above, (S2),(S4) and (S5) hold for $G^{\prime}$. Since all pairs in $N(a)$ and $N\left(b_{1}\right)$ are thin, $G^{\prime}$ is Sperner. The cycle $b_{1} P_{1} y_{3} a y_{2} b_{2} x b_{1}$ certifies that $G^{\prime}$ is 2 -connected.

Thus there is $b_{0} \in N\left(y_{1}\right)-a-b_{1}$. If $G-b_{1}-y_{1}$ has a path $P_{0}$ from $b_{0}$ to $N(a)-y_{1}$, then we have a case similar to $z_{1}=y_{1}$ above (by taking either the path $y_{1} b_{0} P_{0}$ or a portion of it with an endpoint in $P$. Hence there is no such path. But then $G-V(P)-N(a)$ has a $b_{0}, b_{1}$-path $P_{2}$. In this case, we consider $G^{\prime}=G-y_{1} b_{1}$. As above, (S2),(S4) and (S5) hold for $G^{\prime}$. Since all pairs in $N\left(b_{1}\right)$ are thin, $G^{\prime}$ is Sperner. The cycle $y_{1} b_{0} P_{2} b_{1} x b_{2} y_{2} a y_{1}$ certifies that $G^{\prime}$ is 2-connected.

Case 2: $b_{1}$ is happy. Then there is another common neighbor $b_{1}^{\prime}$ of $x$ and $y_{1}$. Again, consider $G^{\prime}=G-a y_{1}$. As above, (S2),(S4) and (S5) hold for $G^{\prime}$. Since all pairs in $N(a)$ are thin, $G^{\prime}$ is Sperner. If $b_{1}^{\prime} \notin P_{1}$ and $z_{2} \in N(a)-y_{t}$ then the cycle $b_{1} P_{1} z_{2} a y_{2} b_{2} x b_{1}^{\prime} y_{1} b_{1}$ certifies that $G^{\prime}$ is 2-connected. If $b_{1}^{\prime} \notin P_{1}$ and $z_{2} \in V(P)$ then the cycle $b_{1} P_{1} z_{2} P\left(z_{2}, a\right) a y_{2} b_{2} x b_{1}^{\prime} y_{1} b_{1}$ does it. If $b_{1}^{\prime} \in P_{1}$, then we switch the roles of $b_{1}$ and $b_{1}^{\prime}$ : consider the path $P_{1}^{\prime}=$ $P_{1}\left(b_{1}^{\prime}, z_{2}\right)$.

So, below we assume $\{x, a\}$ separates $y_{1}$ from the rest of $N(a)$.

Claim 46. Vertex a has only one neighbor (namely, $y_{1}$ ) in the component $C_{1}$ of $G-x-a$ containing $y_{1}$ and $b_{1}$.

Proof. Otherwise, $\{x, a\}$ would not separate $y_{1}$ from $N(a)-y_{1}$. 
Similarly to the definition of $G_{2}$, let $C_{1}$ be the vertex set of the component of $G-a-x$ containing $y_{1}$ and let $G_{1}=G\left[C_{1} \cup\{a, x\}\right]$. By Claim 46, $C_{1} \cap N(a)=\left\{y_{1}\right\}$.

Claim 47. If $G_{1}-a$ is not 2-connected, then $b_{1}$ is unhappy and for some $y \in N\left(b_{1}\right)$, $G-b_{1} y$ satisfies (S1)-(S5). In particular, $G$ admits a shrinking of type (1).

Proof.

Case 1: $G-a-b_{1}$ has an $x, y_{1}$-path $P$. Then $P+b_{1}$ forms a cycle in $G_{1}-a$ containing $x$ and $y_{1}$. Since $G$ is 2-connected and $\left\{y_{1}, x\right\}$ is a separating set in $G_{1}$, this finishes the case.

Case 2: $d\left(b_{1}\right)=2$. Then $y_{1}$ can play the role of $y_{2}$, and we are done by (5).

Case 3: Vertex $b_{1}$ separates $y_{1}$ from $x$ in $G_{1}-a$, and $b_{1}$ has a neighbor $y^{\prime} \notin\left\{x, y_{1}\right\}$. If $b_{1}$ were happy, there would be $b^{\prime} \neq b_{1}$ adjacent to $x$ and $y_{1}$ and we would have Case 1 . So, $b_{1}$ is unhappy. Let $P_{1}$ be a shortest path from $y^{\prime}$ to $\{a, x\}$ in $G-b_{1}$. and $z$ be the last vertex on $P_{1}$.

Suppose first that $z=a$. Then by Claim 46, the second to last vertex of $P_{1}$ is $y_{1}$. Consider $G^{\prime}=G-y_{1} b_{1}$. As above, (S2),(S4) and (S5) hold for $G^{\prime}$. Since $b_{1}$ is unhappy, all pairs in $N\left(b_{1}\right)$ are thin. Thus $G^{\prime}$ is Sperner. The cycle $y^{\prime} P_{1} a y_{2} b_{2} x b_{1} y^{\prime}$ certifies that $G^{\prime}$ is 2 -connected.

Suppose now that $z=x$. Since Case 1 does not hold, $y_{1} \notin P_{1}$. Consider $G^{\prime}=G-x b_{1}$. As above, (S2),(S4) and (S5) hold for $G^{\prime}$. Since all pairs in $N\left(b_{1}\right)$ are thin, $G^{\prime}$ is Sperner. The cycle $y^{\prime} P_{1} x b_{2} y_{2} a y_{1} b_{1} y^{\prime}$ certifies that $G^{\prime}$ is 2-connected.

Claim 48. $G-C_{1}$ and $G-C_{2}$ are 2-connected Sperner $r^{-}$-graphs.

Proof. Let $P$ be a shortest $y_{3}, x$-path in $G-a$. By Claim 44 and $45, P$ avoids $C_{1} \cup C_{2}$. For $i=1,2$, the cycle $y_{3} P x b_{3-i} y_{3-i} a y_{3}$ certifies that $G-C_{i}$ is 2 -connected. Since the degrees of the vertices in $G-C_{1}$ and $G-C_{2}$ are dominated by those in $G, G-C_{1}$ and $G-C_{2}$ are $r^{-}$-graphs. Since $a$ is the only vertex in $A \cap V\left(G-C_{i}\right)$ whose degree decreased w.r.t. $G$ and all pairs in $N(a)$ are thin, $G-C_{1}$ and $G-C_{2}$ are Sperner.

Now set $B_{1}=G_{1}-a, B_{2}=G_{2}-a$, and $x_{1}=x_{2}=x$. Note that the choice of $y_{t}$ in the construction of $G_{t}$ was arbitrary. So we may repeat the proof instead taking $G^{\prime \prime}$ to be the graph obtained by gluing $N(a)-y_{1}$ into a single vertex $y^{* *}$. If $G^{\prime \prime}$ satisfies (S1) - (S5), then we are done. Otherwise we find some vertices $y_{1}^{\prime}, y_{2}^{\prime} \in N(a)-y_{1}$ which play the role of $y_{1}$ and $y_{2}$. We may assume that $y_{1}^{\prime} \notin\left\{y_{1}, y_{2}\right\}$ and it is coupled with some vertex $x^{\prime}$ which plays the role of $x$.

Again, repeating the previous proofs for Claims 41-48 with $y_{1}^{\prime}$ and $y_{2}^{\prime}$, we obtain that either $G$ admits a shrinking, or we can define $G_{1}^{\prime}$ similarly to play the role of $G_{1}$ (defined after Claim 46) for $y_{1}^{\prime}$. Let $B_{3}=G_{1}^{\prime}-a, y_{3}=y_{1}^{\prime}$, and $x_{3}=x^{\prime}$. We now show that (B1)(B5) hold.

(B1) and (B3) are trivial. Since $G$ was Sperner each vertex of $A \cap V\left(B_{i}\right)$ has the sane neighborhood in $B_{i}, B_{i}$ is also Sperner. Hence together with (5) and Claim (47), we get (B2). Claim 48 proves (B4). Claims 44 and 45 imply that $V\left(B_{1}\right) \cap V\left(B_{2}\right)=\{x\}$, and $y_{1}^{\prime}\left(=y_{3}\right)$ is contained in a component of $G-\{a, x\}$ not containing $y_{1}$ and $y_{2}$. In particular, 
$B_{3}$ is disjoint from $B_{1}$ and $B_{2}$ except possibly at $x^{\prime}$ if $x^{\prime}=x$. This proves (B5) and thus Lemma 34.

\subsection{Consequences of Lemma 34}

This technical lemma implies the following more applicable fact.

Lemma 49. Suppose $k \geqslant 5, r \geqslant 3$ are integers with $k \geqslant r$. Set $t=\lfloor(k-1) / 2\rfloor$. Let $G=(A, Y ; E)$ be a Sperner layered 2-connected $r^{-}$-bigraph with $c(G)<2 k$ that is not happy. Then either $G$ admits a shrinking such that the resulting graph satisfies (S1) (S5), or there exists an unhappy vertex $a^{*} \in A$ and corresponding subgraphs $B_{1}^{*}, B_{2}^{*}, B_{3}^{*}$ satisfying (B1) - (B5) such that $B_{1}^{*}$ is happy and $\left|A \cap B_{1}^{*}\right| \leqslant\left(\begin{array}{c}t \\ \min \{r-1,\lfloor t / 2\rfloor\}\end{array}\right)\left(\left|Y \cap B_{1}^{*}\right|-2\right)$.

Proof. Suppose $G$ does not admit any shrinking. By Lemma 34, for each unhappy vertex $a$ we obtain some $\left\{y_{i}, x_{i}, B_{i}\right\}$ for $i \in\{1,2,3\}$ satisfying (B1) - (B5).

Claim 50. For each unhappy $a$, at most one $B_{i}$ has a $\left(x_{i}, y_{i}\right)$-path of length $k$ or longer.

Proof. Suppose without loss of generality that for $i \in\{1,2\}$, there exists a $\left(y_{i}, x_{i}\right)$-path $P_{i}$ in $B_{i}$ of length at least $k$. Recall that $y_{1}, y_{2} \in N(a)$. Let $P_{3}$ be a $\left(x_{1}, x_{2}\right)$-path internally disjoint from $V\left(B_{1}\right) \cup V\left(B_{2}\right)$ (where $P_{3}$ may be a singleton). Then $P_{1} \cup P_{3} \cup P_{2} \cup a$ is a cycle of length at least $2 k-1$, i.e., length at least $2 k$.

Among all vertices in $A$ that are not happy, choose $a$ and a corresponding 2-connected graph $B_{1}$ from Lemma 34 so that (a) $B_{1}$ does not have a $\left(x_{i}, y_{i}\right)$-path of length $k$ or longer, and (b) subject to (a), $\left|V\left(B_{1}\right)\right|$ is minimized.

Suppose first that $B_{1}$ contains an unhappy vertex $a^{\prime}$. By Lemma 34, there exists $\left\{x_{i}^{\prime}, y_{i}^{\prime}, B_{i}^{\prime}\right\}$ for $i \in\{1,2,3\}$ satisfying (B1)-(B5) with $a^{\prime}$.

Claim 51. At most one $j \in\{1,2,3\}$ satisfies $V\left(B_{j}^{\prime}\right) \nsubseteq V\left(B_{1}\right)$.

Proof. Suppose without loss of generality $V\left(B_{2}^{\prime}\right) \nsubseteq V\left(B_{1}\right)$ and $V\left(B_{3}^{\prime}\right) \nsubseteq V\left(B_{1}\right)$. Then since $\left\{x_{1}, a\right\}$ separates $B_{1}$ from $G-\left(B_{1}-x\right)-a$, and $B_{2}^{\prime}$ and $B_{3}^{\prime}$ are 2-connected, $\left\{x_{1}, a\right\} \subseteq$ $V\left(B_{2}^{\prime}\right)$ and $\left\{x_{1}, a\right\} \subseteq V\left(B_{3}^{\prime}\right)$. But this violates (B5).

Therefore we may assume $V\left(B_{1}^{\prime}\right), V\left(B_{2}^{\prime}\right) \subseteq V\left(B_{1}\right)$. By Claim 50, we can also assume that $V\left(B_{1}^{\prime}\right)$ has no $\left(x_{1}^{\prime}, y_{1}^{\prime}\right)$-path of length $k$ or longer. Furthermore, since $a^{\prime} \in V\left(B_{1}\right)-$ $V\left(B_{1}^{\prime}\right),\left|V\left(B_{1}^{\prime}\right)\right|<\left|V\left(B_{1}\right)\right|$. But this contradicts the choice of $a$ and $B_{1}$. Thus $B_{1}$ cannot have any unhappy vertices, i.e., $B_{1}$ is happy.

Consider the shadow $\partial\left(B_{1}\right)$ of $B_{1}$. By Lemma $31, \partial\left(B_{1}\right)$ is not $\lceil(k+1) / 2\rceil$-path connected, otherwise $B_{1}$ would contain an $\left(x_{1}, y_{1}\right)$-path of length at least $2\lceil(k+1) / 2\rceil-1 \geqslant$ $k$, a contradiction.

Let $\alpha=\lceil(k-1) / 2\rceil, \beta=\lfloor(k-1) / 2\rfloor$.

Claim 52. $\frac{1}{\alpha-2}(\underset{\min \{r,\lfloor\alpha / 2\rfloor\}}{\alpha}) \leqslant(\underset{\min \{r-1,\lfloor\beta / 2\rfloor\}}{\beta})$. 
Proof. First suppose $\alpha=\beta$, i.e., $k$ is odd. Then the case $\min \{r,\lfloor\alpha / 2\rfloor\}=\alpha / 2$ is trivial. Otherwise $\frac{1}{\alpha-2}\left(\begin{array}{c}\alpha \\ r\end{array}\right)=\frac{1}{\alpha-2} \frac{\alpha-r+1}{r}\left(\begin{array}{c}\beta \\ r-1\end{array}\right) \leqslant\left(\begin{array}{c}\beta \\ r-1\end{array}\right)$. So assume $\alpha=\beta+1$. If $\min \{r,\lfloor\alpha / 2\rfloor\}=r$ (so $\min \left\{r-1,\lfloor\beta / 2\rfloor=r-1\right.$ ), then we have $\frac{1}{\alpha-2}\left(\begin{array}{c}\alpha \\ r\end{array}\right)=\frac{1}{\beta-1} \frac{\beta+1}{r}\left(\begin{array}{c}\beta \\ r-1\end{array}\right) \leqslant\left(\begin{array}{c}\beta \\ r-1\end{array}\right)$. Otherwise if $\lfloor\alpha / 2\rfloor<r$, then $\lfloor\beta / 2\rfloor \leqslant r-1$, and

$$
\begin{aligned}
\frac{1}{\alpha-2}\left(\begin{array}{c}
\alpha \\
\lfloor\alpha / 2\rfloor
\end{array}\right) & =\frac{1}{\beta-1}\left(\begin{array}{c}
\beta+1 \\
\lfloor(\beta+1) / 2\rfloor
\end{array}\right) \\
& =\frac{1}{\beta-1} \frac{\beta+1}{\lfloor(\beta+1) / 2\rfloor}\left(\begin{array}{c}
\beta \\
\lfloor(\beta+1) / 2\rfloor-1
\end{array}\right) \\
& \leqslant\left(\begin{array}{c}
\beta \\
\lfloor\beta / 2\rfloor
\end{array}\right) .
\end{aligned}
$$

Therefore because $\partial\left(B_{1}\right)$ is not $(\alpha+1)$-path connected, by Theorem 26 and the previous claim,

$$
\begin{aligned}
\left|A \cap B_{1}\right| & \leqslant N_{\mathrm{Sp}}\left(\partial\left(B_{1}\right), r\right) \\
& \leqslant \frac{\left|Y \cap B_{1}\right|-2}{\alpha-2}\left(\begin{array}{c}
\alpha \\
\min \{r,\lfloor\alpha / 2\rfloor\}
\end{array}\right) \\
& \leqslant\left(\left|Y \cap B_{1}\right|-2\right)\left(\begin{array}{c}
\beta \\
\min \{r-1,\lfloor\beta / 2\rfloor\}
\end{array}\right) .
\end{aligned}
$$

\section{Constructing happy $r^{-}$-graphs}

In this section, we translate Lemma 34 into the language of $r^{-}$-graphs. We also refine it.

\subsection{Unhappy $r^{-}$-graphs}

A Sperner $r^{-}$-graph $\mathcal{H}$ is happy if its layered incidence bigraph $I(\mathcal{H})$ is happy, and is unhappy otherwise. The happy and unhappy vertices in $I(\mathcal{H})$ correspond to happy and unhappy edges in $\mathcal{H}$.

For an unhappy edge $e$ in an unhappy $r^{-}$-graph $\mathcal{H}$ and a vertex $v \in e$, let $F(\mathcal{H}, e, v)$ denote the $r^{-}$-graph obtained from $\mathcal{H}$ by replacing $e$ with $e-v$.

A vertex $v$ of degree 2 in an unhappy $r^{-}$-graph $\mathcal{H}$ is special if each of the two incident edges, say $e_{1}$ and $e_{2}$, is either unhappy or a graph edge (i.e., contains exactly two vertices). If $v$ is special and incident with $e_{1}$ and $e_{2}$, then $F\left(\mathcal{H}, v, e_{1}, e_{2}\right)$ is the $r^{-}$-graph obtained from $\mathcal{H}$ by deleting $v$ and for $i=1,2$ deleting $e_{i}$ if $\left|e_{i}\right|=2$ and replacing $e_{i}$ with $e_{i}-v$ otherwise.

A graph edge $v u$ in an unhappy $r^{-}$-graph $\mathcal{H}$ is special if both $v$ and $u$ are special, and both adjacent to $v u$ edges are unhappy. If $v u$ is special and adjacent to $e_{1}$ and $e_{2}$, then $F(\mathcal{H}, v u)$ is the $r^{-}$-graph obtained from $\mathcal{H}$ by deleting $v$ and $u$, replacing $e_{1}$ with $e_{1}-v$, and replacing $e_{2}$ with $e_{2}-u$.

A 2 -block in a 2-connected $\mathcal{H}$ is a 2-connected $\mathcal{H}^{\prime} \subset \mathcal{H}$ such that only two vertices of $\mathcal{H}^{\prime}$ have neighbors outside of $\mathcal{H}^{\prime}$. These two vertices will be called outer vertices of $\mathcal{H}^{\prime}$. 
A 2-block $\mathcal{H}^{\prime}$ with outer vertices $x$ and $y$ in an unhappy Sperner $r^{-}$-graph $\mathcal{H}$ is special if

$\mathcal{H}^{\prime}$ is happy and there is exactly one edge, say $a$, in $G-E\left(\mathcal{H}^{\prime}\right)$ containing $y$, and this edge does not contain $x$.

Given a special 2-block $\mathcal{H}^{\prime}$ with outer vertices $x$ and $y$ in an unhappy Sperner $r^{-}$-graph $\mathcal{H}$, the $r^{-}$-graph $F\left(\mathcal{H}, \mathcal{H}^{\prime}, x, y\right)$ is obtained from $\mathcal{H}$ by deleting all vertices of $\mathcal{H}^{\prime}-x-y$ together with the edges containing them and adding edge $\{x, y\}$ if it is not in $\mathcal{H}$.

Translating from the language of incidence bipartite graphs to hypergraphs, we obtain the following versions of Lemmas 30 and 31 about Berge cycles and Berge paths.

Lemma 53. Let $r \geqslant 3$. Let $\mathcal{H}$ be a happy $r^{-}$-graph. If the 2-shadow $\partial_{2} \mathcal{H}$ contains a cycle of length $\ell \geqslant r+1$, then $\mathcal{H}$ contains a Berge cycle of length $\ell$ on the same base vertices. Furthermore, if $\partial_{2} \mathcal{H}$ contains a path, then $\mathcal{H}$ contains a Berge path with the same base vertices.

For simplicity, for an $r^{-}$-graph $\mathcal{H}$, denote $\sum|E(\mathcal{H})|:=\sum_{e \in E(\mathcal{H})}|e|$. For example, if $\mathcal{H}$ is $r$-uniform, then $\sum|E(\mathcal{H})|=r|E(\mathcal{H})|$. We also obtain the following as a corollary of Lemma 34 (particularly following Claims 35-47) and Lemma 49.

Lemma 54. Suppose $k \geqslant r \geqslant 3$ are integers, and set $t=\lfloor(k-1) / 2\rfloor$. Let $\mathcal{H}$ be a Sperner 2 -connected $r^{-}$-graph with $c(\mathcal{H})<k$ that is not happy. Then we can obtain a Sperner 2-connected $r^{-}$-graph $\mathcal{H}^{\prime}$ such that

(i) $\sum\left|E\left(\mathcal{H}^{\prime}\right)\right| \leqslant \sum|E(\mathcal{H})|,\left|V\left(\mathcal{H}^{\prime}\right)\right| \leqslant|V(\mathcal{H})|$, and $\sum\left|E\left(\mathcal{H}^{\prime}\right)\right|+\left|V\left(\mathcal{H}^{\prime}\right)\right|<\sum|E(\mathcal{H})|+$ $|V(\mathcal{H})|$;

(ii) $|E(\mathcal{H})|-\left|E\left(\mathcal{H}^{\prime}\right)\right| \leqslant\left(\begin{array}{c}t \\ \min \{r-1,\lfloor t / 2\rfloor\}\end{array}\right)\left(|V(\mathcal{H})|-\left|V\left(\mathcal{H}^{\prime}\right)\right|\right)$; and

(iii) $c\left(\mathcal{H}^{\prime}\right)<k$

using one of the following transformations:

(T1) for an unhappy edge $e$ and $v \in e$, replacing $H$ with $F(H, e, v)$;

(T2) for a special vertex $v$ with incident edges $e_{1}$ and $e_{2}$, replace $H$ with $F\left(H, v, e_{1}, e_{2}\right)$;

(T3) for a special edge vu, replace $H$ with $F(H, v u)$;

(T4) glue together all but one vertices of an unhappy edge;

(T5) for a special 2-block $H^{\prime}$ with outer vertices say $x, y$, replace $H$ with $F\left(H, H^{\prime}, x, y\right)$.

Furthermore, if (T5) is not applied, then instead of (ii), we obtain $|E(\mathcal{H})|-\left|E\left(\mathcal{H}^{\prime}\right)\right| \leqslant$ $\left(|V(\mathcal{H})|-\left|V\left(\mathcal{H}^{\prime}\right)\right|\right)$. 


\subsection{A refinement of Lemma 54}

Suppose we start from a Sperner 2-connected unhappy $r^{-}$-graph $\mathcal{H}$ with at least $k$ vertices and $c(\mathcal{H})<k$. Lemma 54 provides that we can obtain from $\mathcal{H}$ a happy Sperner 2connected $r^{-}$-graph in several steps using the following rule at each step:

$$
\text { if possible, apply (T1); if not then try (T2), then (T3) and so on. }
$$

We may think that we have started from $\mathcal{H}=\mathcal{H}_{0}$ and after Step $i$ obtain $\mathcal{H}_{i}$ from $\mathcal{H}_{i-1}$ using one of (T1)-(T5).

Claims 36- 37 in the proof of Lemma 54 yield that following Rule (6), at each Step $i$, if (T1) is not applied on Step $i+1$, then in each unhappy edge a of $\mathcal{H}_{i}$, thick pairs are disjoint,

and

if neither (T1) nor (T2) is applied on Step $i+1$, then all pairs of vertices in each unhappy edge a of $\mathcal{H}_{i}$ are thin.

Claim 55. If (T2) was applied on Step $i$, then (T1) cannot be applied on Step $i+1$.

Proof. Suppose $\mathcal{H}_{i}=F\left(\mathcal{H}_{i-1}, v, e_{1}, e_{2}\right)$ and $\mathcal{H}_{i+1}=F\left(\mathcal{H}_{i}, e_{0}, w\right)$.

Case 1: Edge $e_{0}$ is neither $e_{1}-v$ nor $e_{2}-v$. We want to show that in this case, $e_{0}$ is unhappy in $\mathcal{H}_{i-1}$ and $\mathcal{H}^{\prime}=F\left(\mathcal{H}_{i-1}, e_{0}, w\right)$ is a Sperner 2-connected $r^{-}$-graph satisfying (i)-(iii) with $\mathcal{H}_{i-1}$ in place of $\mathcal{H}$. That would contradict Rule (6).

To prove the first part (that $e_{0}$ is unhappy in $\mathcal{H}_{i-1}$ ), recall that $e_{0}$ is unhappy in $\mathcal{H}_{i}$. But the codegree in $\mathcal{H}_{i}$ of each pair in $V\left(\mathcal{H}_{i}\right)$ is the same as in $\mathcal{H}_{i-1}$.

To prove the second part, we use the fact that $\mathcal{H}^{\prime}$ can be obtained from $\mathcal{H}_{i+1}$ by adding back vertex $v$ and for $j=1,2$ constructing $e_{j}$ either by adding $v$ to $e_{j}-v \in \mathcal{H}_{i+1}$ when $\left|e_{j}\right| \geqslant 3$ or adding edge $e_{j}$ when $\left|e_{j}\right|=2$. Since the incidence graph $I\left(\mathcal{H}_{i+1}\right)$ is 2-connected and this operation corresponds to adding a vertex of degree 2 or an ear to $I\left(\mathcal{H}_{i+1}\right), I\left(\mathcal{H}^{\prime}\right)$ also is 2-connected. Since $\mathcal{H}_{i+1}$ is Sperner, and $\mathcal{H}^{\prime}$ differs from it only $e_{1}, e_{2}$ and $v, H^{\prime}$ is also Sperner: new edges are not contained in any old edge because of $v$, and no old edge can be contained in $e_{j}$, since otherwise it would be contained in $e_{j}-v$ in $\mathcal{H}_{i+1}$. Properties (i)-(iii) are trivial.

Case 2: $e_{0}=e_{1}-v$. In this case, we know that $e_{1}$ is unhappy in $\mathcal{H}_{i-1}$ and want to show that $\mathcal{H}^{\prime}=F\left(\mathcal{H}_{i-1}, e_{1}, w\right)$ is a Sperner 2-connected $r^{-}$-graph satisfying (i)-(iii) with $\mathcal{H}_{i-1}$ in place of $\mathcal{H}$. Now $\mathcal{H}^{\prime}$ can be obtained from $\mathcal{H}_{i+1}$ by adding back vertex $v$, adding $v$ to $e_{0}-w$ and constructing $e_{2}$ either by adding $v$ to $e_{2}-v \in \mathcal{H}_{i+1}$ when $\left|e_{2}\right| \geqslant 3$ or adding edge $e_{2}$ when $\left|e_{2}\right|=2$. The rest is as in Case 1.

Practically the same proof yields the following similar claim.

Claim 56. If (T3) was applied on Step $i$, then (T1) cannot be applied on Step $i+1$.

The proof of the next claim is somewhat different. 
Claim 57. If (T4) was applied on Step i, then (T1) cannot be applied on Step $i+1$.

Proof. Suppose $\mathcal{H}_{i-1}$ has an unhappy edge $a=\left\{y_{1}, \ldots, y_{t}\right\}$ such that $\mathcal{H}_{i}$ is obtained from $H_{i-1}$ by gluing $\left\{y_{1}, \ldots, y_{t-1}\right\}$ into a new vertex $y^{*}$, and $\mathcal{H}_{i+1}=F\left(\mathcal{H}_{i}, e, w\right)$. By (8), all pairs of vertices in each unhappy edge of $\mathcal{H}_{i-1}$ are thin. In particular, the size of each edge in $\mathcal{H}_{i}$ apart from the edge $y^{*} y_{t}$ is the same as in $\mathcal{H}_{i-1}$.

Case 1: $w \neq y^{*}$. By (9), in $\mathcal{H}_{i-1},|e \cap a| \leqslant 1$. So, since $e$ is unhappy in $\mathcal{H}_{i}$, it is also unhappy in $\mathcal{H}_{i-1}$. We want to show that $\mathcal{H}^{\prime}=F\left(\mathcal{H}_{i-1}, e, w\right)$ is a Sperner 2-connected $r^{-}$-graph satisfying (i)-(iii). Since each pair in $e$ is thin, $\mathcal{H}^{\prime}$ is Sperner. Properties (i)-(iii) are evident, so we need to check that $\mathcal{H}^{\prime}$ is 2-connected.

By construction, $\mathcal{H}^{\prime}$ can be obtained from the 2-connected $\mathcal{H}_{i+1}$ by blowing up vertex $y^{*}$ into vertices $y_{1}, \ldots, y_{t-1}$ (each of a positive degree) and replacing edge $y^{*} y_{t}$ with $a$. In terms of the incidence graphs, in the 2-connected $I\left(\mathcal{H}_{i+1}\right)$, we split $y^{*}$ into $t-1$ vertices of degree at least 1 , delete vertex $y^{*}$ and add vertex $a$ adjacent to $y_{1}, \ldots, y_{t}$. It is easy to check that the new graph is 2-connected.

Case 2: $w=y^{*}$. By (9), there is a unique $v_{1} \in a-y_{t}$ such that $e^{\prime}=e-y^{*}+v_{1} \in \mathcal{H}_{i-1}$. Since $e$ is unhappy in $\mathcal{H}_{i}$, it has a pair $x y$ of codegree at most $|e|-2$. If $y^{*} \notin\{x, y\}$, then the codegree of $x y$ in $\mathcal{H}_{i-1}$ also is at most $|e|-2$. And if $y^{*}=y$, then the codegree of $y_{1} x$ in $\mathcal{H}_{i-1}$ is at most $|e|-2$. Thus $e^{\prime}$ is unhappy in $\mathcal{H}_{i-1}$. The rest is as in Case 1.

\subsection{Stopping at $k-1$ vertices}

Lemma 58. Suppose $r \geqslant 3$ and $k \geqslant r$ are integers. Let $\mathcal{H}$ be a Sperner 2-connected $r^{-}$graph with $|V(\mathcal{H})| \geqslant k$ and $c(\mathcal{H})<k$ that is not happy. Suppose $\mathcal{H}=\mathcal{H}_{0}, \ldots, \mathcal{H}_{i}, \mathcal{H}_{i+1}$ is a sequence of $r^{-}$-graphs obtained by iteratively applying Lemma 54 following Rule (6) to $\mathcal{H}$ until $\mathcal{H}_{i+1}$ is happy. If (T5) was never applied and $\left|V\left(\mathcal{H}_{i+1}\right)\right|=k-1$, then $\left|E\left(\mathcal{H}_{i+1}\right)\right| \leqslant$ $\left(\begin{array}{c}k-2 \\ \min \{r,\lfloor(k-2) / 2\rfloor\}\end{array}\right)+2$.

Proof. Since (T1) does not change the number of vertices and $\mathcal{H}_{0}$ has at least $k$ vertices, one of (T2), (T3), or (T4) was applied. Moreover, by Claims 55-57, one of (T2), (T3), or (T4) was applied to $\mathcal{H}_{i}$ to obtain the happy $r^{-}$-graph $\mathcal{H}_{i+1}$. For short, denote $\mathcal{H}^{\prime}=\mathcal{H}_{i+1}$.

If $\mathcal{H}^{\prime}$ has a vertex of degree at most 3 , then the number of edges in $\mathcal{H}^{\prime}$ is at most $\left(\begin{array}{c}k-2 \\ \min \{r,\lfloor(k-2) / 2\rfloor\}\end{array}\right)+\left(\begin{array}{c}2 \\ \min \{r-1,1\}\end{array}\right)$, and we are done. Hence

$$
\delta\left(\mathcal{H}^{\prime}\right) \geqslant 3
$$

In the following, for any $r^{-}$-graph $\mathcal{A}$ and any vertex $v \in V(\mathcal{A})$, we use $\mathcal{A}-v$ to denote the $r^{-}$-graph obtained by removing vertex $v$ and shrinking any edge $e$ that contains $v$ to the edge $e-v$, unless $|e|=2$, in which case we simply delete $e$ in $\mathcal{A}-v$. Note that $\mathcal{A}-v$ need not be Sperner, even if $\mathcal{A}$ is Sperner.

Case 1: (T4) was the last applied operation. Let $a=\left\{y_{1}, \ldots, y_{t}\right\}$ be the unhappy edge such that $\mathcal{H}^{\prime}$ is obtained from $H_{i}$ by gluing $\left\{y_{1}, \ldots, y_{t-1}\right\}$ into a new vertex $y^{*}$. Since 
$\mathcal{H}^{\prime}$ is happy, $\mathcal{H}_{i}-a$ is happy. The $r^{-}$-graph $F\left(\mathcal{H}_{i}, a, y_{t}\right)$ satisfies (i)-(iii) and is Sperner by (9). So if $F\left(\mathcal{H}^{\prime}, a, y_{t}\right)$ is 2 -connected, then we would have applied (T1) to $\mathcal{H}_{i}$ instead of (T4), a contradiction to Rule (6). Therefore

the incidence graph $I\left(\mathcal{H}_{i}-a\right)$ has a vertex $x_{t}$ separating $y_{t}$ from $\left\{y_{1}, \ldots, y_{t-1}\right\}$.

If $x_{t}$ corresponds to an edge $b$ in $\mathcal{H}_{i}-a$, then some pair of its vertices is thin. So, since $\mathcal{H}_{i}-a$ is happy, $|b|=2$. Then instead of $x_{t}$, we can choose as a vertex $x_{t}^{\prime}$ separating $y_{t}$ from $\left\{y_{1}, \ldots, y_{t-1}\right\}$ the neighbor of $x_{t}$ that is farther from $y_{t}$. Thus we may assume that $x_{t}$ corresponds to a vertex in $\mathcal{H}_{i}-a$.

If $x_{t} \notin\left\{y_{1}, \ldots, y_{t-1}\right\}$, then $y_{t}$ and $y^{*}$ are also separated by $x_{t}$ in $\mathcal{H}^{\prime}-y^{*} y_{t}$. Since there are at least 2 components in $\mathcal{H}^{\prime}-y^{*} y_{t}-x_{t}$, the largest block of $\mathcal{H}^{\prime}-y^{*} y_{t}$ has at most $\left|V\left(\mathcal{H}^{\prime}\right)-1\right|=k-2$ vertices.

We have that

$\left|E\left(\mathcal{H}^{\prime}\right)\right|=\left|E\left(\mathcal{H}^{\prime}-y^{*} y_{t}\right)\right|+1 \leqslant\left(\begin{array}{c}k-2 \\ \min \{r,\lfloor(k-2) / 2\rfloor\}\end{array}\right)+1+1=\left(\begin{array}{c}k-2 \\ \min \{r,\lfloor(k-2) / 2\rfloor\}\end{array}\right)+2$.

If $x_{t} \in\left\{y_{1}, \ldots, y_{t-1}\right\}$, then let $\mathcal{C}$ be a component of $\left(\mathcal{H}_{i}-a\right)-x_{t}$ which does not contain $y_{t}$. Then $\mathcal{C}$ contains a vertex $y \notin\left\{y_{1}, \ldots, y_{t-1}\right\}$, otherwise every edge of $\mathcal{C}+x_{t}$ in $\mathcal{H}_{i}$ would be a subset of the edge $a$, contradicting that $\mathcal{H}_{i}$ is Sperner. Thus in $\mathcal{H}^{\prime}-y^{*} y_{t}$, $y$ and $y_{t}$ are in different blocks. Hence we again get $\left|E\left(\mathcal{H}^{\prime}\right)\right| \leqslant\left(\begin{array}{c}k-2 \\ \min \{r,\lfloor(k-2) / 2\rfloor\}\end{array}\right)+2$.

Case 2: $\mathcal{H}_{i+1}=F\left(\mathcal{H}_{i}, v, e_{1}, e_{2}\right)$ for some special vertex $v$. By $(7)$, if $\left|e_{1}\right| \geqslant 4$, then some pair in $e_{1}-v$ is thin, and hence $e_{1}-v$ is unhappy in $\mathcal{H}_{i+1}$, a contradiction the happiness of $\mathcal{H}_{i+1}$. Thus $\left|e_{1}\right|,\left|e_{2}\right| \leqslant 3$. Since $\mathcal{H}_{i}$ was unhappy, we may assume that $\left|e_{1}\right|=3$, say $e_{1}=\left\{v, v^{\prime}, v^{\prime \prime}\right\}$. By $(7)$, either $v v^{\prime}$ or $v v^{\prime \prime}$ is a thin pair in $\mathcal{H}_{i}$. Suppose $v v^{\prime \prime}$ is thin. Consider $\mathcal{H}^{\prime \prime}=F\left(\mathcal{H}_{i}, e_{1}, v^{\prime}\right)$. Since $v v^{\prime \prime}$ is thin, $\mathcal{H}^{\prime \prime}$ is Sperner. If $\mathcal{H}^{\prime \prime}$ is 2-connected, we get a contradiction to Rule (6). Thus the incidence graph $I\left(\mathcal{H}^{\prime \prime}\right)$ has a cut vertex $x$ separating $v^{\prime}$ from $\left\{v, v^{\prime \prime}\right\}$. We claim that

we can choose $x$ corresponding to a vertex in $\mathcal{H}^{\prime \prime}$ distinct from $v$. (We allow $x=v^{\prime \prime}$.)

Indeed, if $v$ separates $v^{\prime}$ from $v^{\prime \prime}$ in $I\left(\mathcal{H}^{\prime \prime}\right)$, then vertex $e_{1}$ in the incidence graph $I\left(\mathcal{H}_{i}\right)$ separates $v^{\prime}$ from $v^{\prime \prime}$, a contradiction to the 2-connectedness of $\mathcal{H}_{i}$. If $x$ corresponds to an edge in $I\left(\mathcal{H}_{i}\right)$, then again $x$ contains thin pairs. If $|x| \geqslant 3$. Then $x$ is unhappy. By the choice $\mathcal{H}_{i+1}$, the only unhappy edge in $\mathcal{H}^{\prime \prime}$ could be $e_{2}$. Recall that in this case, $\left|e_{2}\right|=3$, say $x=e_{2}=\left\{v, w, w^{\prime}\right\}$. But in this case, one of $v, w$ and $w^{\prime}$ also separates $v^{\prime}$ from $v^{\prime \prime}$, and we know that it is not $v$. Recall that $v v^{\prime \prime}$ is a thin pair, and so $v^{\prime \prime} \notin\left\{w, w^{\prime}\right\}$. Otherwise if $|x|=2$, then both of its vertices are cut vertices. This proves (12).

Recall that $\left|V\left(\mathcal{H}^{\prime \prime}\right)\right|=\left|V\left(\mathcal{H}_{i}\right)\right|=k$ and $e\left(\mathcal{H}^{\prime \prime}\right)=e\left(\mathcal{H}_{i}\right) \leqslant e\left(\mathcal{H}_{i+1}\right)+1$. Suppose first that each component of $\mathcal{H}^{\prime \prime}-x$ has at least 3 vertices. Since $\mathcal{H}^{\prime \prime}-x$ has $k-1$ vertices and at least 2 connected components, $k \geqslant 7$, and the largest component of $\mathcal{H}^{\prime \prime}-x$ has at most $k-4$ vertices. Therefore we obtain

$$
e\left(\mathcal{H}_{i+1}\right) \leqslant e\left(\mathcal{H}^{\prime \prime}\right) \leqslant\left(\begin{array}{c}
k-3 \\
\min \{r,\lfloor(k-3) / 2\rfloor\}
\end{array}\right)+\left(\begin{array}{l}
4 \\
2
\end{array}\right) \leqslant\left(\begin{array}{c}
k-2 \\
\min \{r,\lfloor(k-2) / 2\rfloor\}
\end{array}\right)+2 .
$$


Now suppose that some component $\mathcal{C}$ of $\mathcal{H}^{\prime \prime}-x$ contains at most 2 vertices. By (10), $|\mathcal{C}|=2$ and each of the two vertices in $\mathcal{C}$ either has degree in $\mathcal{H}^{\prime \prime}$ less than in $\mathcal{H}_{i+1}$ or is $v$. But the only vertex having degree in $\mathcal{H}^{\prime \prime}$ less than in $\mathcal{H}_{i+1}$ is $v^{\prime}$, and the vertices $v$ and $v^{\prime}$ are in distinct components of $\mathcal{H}^{\prime \prime}-x$.

Case 3: $\mathcal{H}_{i+1}=F\left(\mathcal{H}_{i}, v u\right)$ for some special edge $v u$. Let $e_{1}$ be the unhappy edge incident to $v$ and $e_{2}$ be the unhappy edge incident to $u$. By (8), all pairs in $e_{1}$ and $e_{2}$ are thin. So since $\mathcal{H}_{i+1}$ is happy, $\left|e_{1}\right|=\left|e_{2}\right|=3$. Let $e_{1}=\left\{v, v^{\prime}, v^{\prime \prime}\right\}$ and $e_{2}=\left\{u, u^{\prime}, u^{\prime \prime}\right\}$, where possibly $v^{\prime}=u^{\prime}$. As in Case 2, consider $\mathcal{H}^{\prime \prime}=F\left(\mathcal{H}_{i}, e_{1}, v^{\prime}\right)$. Since $v v^{\prime \prime}$ is thin, $\mathcal{H}^{\prime \prime}$ is Sperner. If $\mathcal{H}^{\prime \prime}$ is 2 -connected, we get a contradiction to Rule (6). Thus the incidence graph $I\left(\mathcal{H}^{\prime \prime}\right)$ has a cut vertex $x$ separating $v^{\prime}$ from $\left\{v, v^{\prime \prime}\right\}$.

Similarly to the proof of (12), we derive

we can choose $x$ corresponding to a vertex in $\mathcal{H}^{\prime \prime}$ distinct from $v$ and $u$. (We allow $x=v^{\prime \prime}$.)

Furthermore, $x \notin\left\{u^{\prime}, u^{\prime \prime}\right\}$. Now $\left|V\left(\mathcal{H}^{\prime \prime}\right)\right|=\left|V\left(\mathcal{H}_{i}\right)\right|=k+1$ and $e\left(\mathcal{H}^{\prime \prime}\right)=e\left(\mathcal{H}_{i}\right)=$ $e\left(\mathcal{H}_{i+1}\right)+1$.

Note that there cannot be any isolated vertices in $\mathcal{H}^{\prime \prime}-x$ since by $(10), \delta\left(\mathcal{H}^{\prime \prime}\right) \geqslant 3$. Also, as in the previous case, there cannot be a component of $\mathcal{H}^{\prime \prime}-x$ with exactly 2 vertices. So we may assume that each component of $\mathcal{H}^{\prime \prime}-x$ has at least 3 vertices.

Let $\mathcal{C}$ be the component of $\mathcal{H}^{\prime \prime}-x$ that contains $v$. Then $\mathcal{C}$ must also contain $u$ and at least two of the vertices in $\left\{v^{\prime \prime}, u^{\prime}, u^{\prime \prime}\right\}$. Therefore $|\mathcal{C}| \geqslant 4$. In particular, since $\mathcal{H}^{\prime \prime}-x$ contains exactly $k$ vertices and at least 2 connected components, $k \geqslant|\mathcal{C}|+3 \geqslant 7$.

As in Case 2, if the largest component of $\mathcal{H}^{\prime \prime}-x$ has at most $k-4$ vertices (so $k \geqslant 8$ since $|\mathcal{C}| \geqslant 4$ ), then

$$
e\left(\mathcal{H}_{i+1}\right) \leqslant e\left(\mathcal{H}^{\prime \prime}\right) \leqslant\left(\begin{array}{c}
k-3 \\
\min \{r,\lfloor(k-3) / 2\rfloor\}
\end{array}\right)+\left(\begin{array}{l}
5 \\
2
\end{array}\right) \leqslant\left(\begin{array}{c}
k-2 \\
\min \{r,\lfloor(k-2) / 2\rfloor\}
\end{array}\right)+2,
$$

a contradiction.

Now suppose a component $\mathcal{C}^{\prime}$ of $\mathcal{H}^{\prime \prime}-x$ has $k-3$ or $k-2$ vertices. If $\mathcal{C}^{\prime}$ contains $v$, (i.e., $\mathcal{C}^{\prime}=\mathcal{C}$ ), then since $\mathcal{C}$ contains $u$ as well, and $u$ and $v$ are incident to exactly 3 edges $\left(v u, e_{1}\right.$, and $\left.e_{2}\right)$,

$$
e\left(\mathcal{H}^{\prime \prime}[\mathcal{C}+x]\right) \leqslant\left(\begin{array}{c}
\left|\mathcal{C}^{\prime}\right|-2+1 \\
\min \left\{r,\left\lfloor\left(\left|\mathcal{C}^{\prime}\right|-2+1\right) / 2\right\rfloor\right\}
\end{array}\right)+3 .
$$

For $\left|\mathcal{C}^{\prime}\right|=k-3$ we get

$$
e\left(\mathcal{H}^{\prime \prime}\right) \leqslant\left(\begin{array}{c}
k-4 \\
\min \{r,\lfloor(k-3) / 2\rfloor\}
\end{array}\right)+3+\left(\begin{array}{l}
4 \\
2
\end{array}\right) \leqslant\left(\begin{array}{c}
k-2 \\
\min \{r,\lfloor(k-2) / 2\rfloor\}
\end{array}\right)+2,
$$

and for $\left|\mathcal{C}^{\prime}\right|=k-2$ we get

$$
e\left(\mathcal{H}^{\prime \prime}\right) \leqslant\left(\begin{array}{c}
k-3 \\
\min \{r,\lfloor(k-3) / 2\rfloor\}
\end{array}\right)+3+\left(\begin{array}{l}
3 \\
2
\end{array}\right) \leqslant\left(\begin{array}{c}
k-2 \\
\min \{r,\lfloor(k-2) / 2\rfloor\}
\end{array}\right)+2 .
$$

So $\mathcal{C}^{\prime} \neq \mathcal{C}$. But since $|\mathcal{C}| \geqslant 4$, we have $\left|V\left(\mathcal{H}^{\prime \prime}\right)\right| \geqslant\left|\mathcal{C}^{\prime}\right|+|\mathcal{C}|+1 \geqslant 4+(k-3)+1=k+2$, a contradiction. 


\section{Proof of Theorem 14}

Proof. Apply Lemma 54 repeatedly to $\mathcal{H}$ following Rule (6) to obtain an $r^{-}$-hypergraph $\mathcal{H}^{\prime}$ that is happy. By Lemma $53, \partial_{2} \mathcal{H}^{\prime}$ has no cycle of length $k$ or longer. Note that every 2 -connected graph has a cycle of length at least 4 , so we may assume $k \geqslant 5$. Also, because $\mathcal{H}^{\prime}$ is a 2-connected hypergraph, $\partial_{2} \mathcal{H}^{\prime}$ is a 2-connected graph.

Set $t=\lfloor(k-1) / 2\rfloor$, and let $n_{S}$ and $m_{S}$ be the number of vertices and $r^{-}$-edges respectively that were deleted going from $\mathcal{H}$ to $\mathcal{H}^{\prime}$ by applying operations (T1)-(T4), and let $n_{B}$ and $m_{B}$ be the number of vertices and $r^{-}$-edges respectively that were deleted from applying operation (T5). So $n=\left|V\left(\mathcal{H}^{\prime}\right)\right|+n_{S}+n_{B}$ and $|E(\mathcal{H})| \leqslant N_{\mathrm{Sp}}\left(\partial_{2} \mathcal{H}^{\prime}, r\right)+m_{S}+m_{B}$. If $\left|V\left(\mathcal{H}^{\prime}\right)\right| \geqslant k$, then by Theorem 21 (applied to $\partial_{2} \mathcal{H}^{\prime}$ ) and Lemma 53, we have

$$
\begin{gathered}
|E(\mathcal{H})| \leqslant N_{\mathrm{Sp}}\left(\partial_{2} \mathcal{H}^{\prime}, r\right)+m_{S}+m_{S} \\
\leqslant \max \left\{f\left(\left|V\left(\mathcal{H}^{\prime}\right)\right|, k, r, 2\right), f\left(\left|V\left(\mathcal{H}^{\prime}\right)\right|, k, r, t\right)\right\}+n_{S}+\left(\begin{array}{c}
t \\
\min \{r-1,\lfloor t / 2\rfloor\}
\end{array}\right) n_{B}
\end{gathered}
$$

First suppose that $n_{B}=0$, i.e., (T5) was never applied. Examining the coefficient of $n_{S}$ we see $1 \leqslant \min \left\{2,\left(\begin{array}{c}t \\ \min \{r-1,\lfloor t / 2\rfloor\}\end{array}\right)\right\}$. So in the case $\left|V\left(\mathcal{H}^{\prime}\right)\right| \geqslant k$, from (14), we get

$$
|E(\mathcal{H})| \leqslant \max \{f(n, r, k, 2), f(n, r, k, t)\},
$$

as desired. Otherwise, if $\left|V\left(\mathcal{H}^{\prime}\right)\right| \leqslant k-1$, then either

$$
\left|E\left(\mathcal{H}^{\prime}\right)\right| \leqslant\left(\begin{array}{c}
k-2 \\
\min \{r,\lfloor(k-2) / 2\rfloor\}
\end{array}\right)+2=f(k-1, k, r, 2)
$$

by Lemma 58 , or $\left|V\left(\mathcal{H}^{\prime}\right)\right| \leqslant k-2$ and

$$
\left|E\left(\mathcal{H}^{\prime}\right)\right| \leqslant\left(\begin{array}{c}
\left|V\left(\mathcal{H}^{\prime}\right)\right| \\
\min \left\{r,\left\lfloor\left|V\left(\mathcal{H}^{\prime}\right)\right| / 2\right\rfloor\right\}
\end{array}\right) \leqslant f\left(\left|V\left(\mathcal{H}^{\prime}\right)\right|, k, r, 2\right) .
$$

Either way we obtain $|E(\mathcal{H})| \leqslant f(n, k, r, 2)$.

So we may assume that at least one application of (T5) was required to obtain $\mathcal{H}^{\prime}$.

Denote $H^{\prime}:=\partial_{2} \mathcal{H}^{\prime}$. Let $Q$ be the $t$-core of $H^{\prime}$ (that is, the resulting graph from applying $t$-disintegration to $\left.H^{\prime}\right)$. If $H^{\prime}$ is $t$-disintegrable, i.e., $Q$ is empty, then $N_{\mathrm{Sp}}\left(H^{\prime}, r\right) \leqslant$ $f\left(\left|V\left(H^{\prime}\right)\right|, k, r, t\right)$ and so by $(14)$, we get $|E(\mathcal{H})| \leqslant f(n, k, r, t)$. So we may assume that $Q$ is non-empty. In particular, since $\delta(Q) \geqslant t+1,|V(Q)| \geqslant t+2$.

Claim 59. The graph $Q$ is 1-hamiltonian.

Proof. First note that $|V(Q)| \leqslant k-1$ : the case for $\left|V\left(H^{\prime}\right)\right| \leqslant k-1$ is trivial, and if $\left|V\left(H^{\prime}\right)\right| \geqslant k$, then by applying Kopylov's Theorem (Theorem 23), we obtain $|V(Q)| \leqslant$ $k-2$.

Next, we claim that $Q$ is 3 -connected. If not, then there exists a cut set $\{x, y\} \subset V(Q)$ and at least two components in $H^{\prime}-\{x, y\}$. Since $\delta(Q) \geqslant t+1$, for each of these 
components $C,|C \cup\{x, y\}| \geqslant t+2$. Hence $|V(Q)| \geqslant 2(t+2)-2 \geqslant k$, a contradiction to $|V(Q)| \leqslant k-1$.

Therefore $Q$ is 3-connected. By Enomoto's Theorem (Theorem 24), $Q$ is $s$-path connected where $s=\min \{|V(Q)|, 2(t+1)\}=|V(Q)|$. I.e., $Q$ is 1-hamiltonian.

Let $q:=|V(Q)|$. Let $\mathcal{B}$ be a special (in particular, happy) block that was removed in some application of $(\mathrm{T} 5)$, and set $B=\partial_{2} \mathcal{B}$. Let $x_{B}$ and $a_{B}$ be the vertex-edge cut pair corresponding to $\mathcal{B}$, where some vertex $y_{B} \in V(\mathcal{B}) \backslash V\left(\mathcal{H}^{\prime}\right)$ is contained in $a_{B}$.

Claim 60. Suppose $H^{\prime}$ is s-path connected. There does not exist a $\left(x_{B}, y_{B}\right)$-path of length at least $k-s+1$ in $B$.

Proof. Since $\mathcal{H}$ is 2-connected, its incidence bigraph contains two shortest disjoint paths $P_{1}, P_{2}$ from $\left\{x_{B}, a_{B}\right\}$ to $V\left(\mathcal{H}^{\prime}\right)$ (where possibly $\left|V\left(P_{1}\right)\right|=1$ or $\left|V\left(P_{2}\right)\right|=1$ ). Note that these paths are internally disjoint from $V\left(\mathcal{H}^{\prime}\right) \cup V(\mathcal{B})$. In $\mathcal{H}, P_{1}$ and $P_{2}$ yield Berge paths $\mathcal{P}_{1}$ and $a \cup \mathcal{P}_{2}$ from $x_{B}$ to $V\left(\mathcal{H}^{\prime}\right)$ and $y_{B}$ to $V\left(\mathcal{H}^{\prime}\right)$ respectively. Say $P_{i}$ has endpoint $v_{i} \in V\left(\mathcal{H}^{\prime}\right)$.

Now suppose there exists a path of length at least $k-s+1$ from $x_{B}$ to $y_{B}$. By Lemma 31 , this yields a Berge path $\mathcal{P}_{3}$ from $x_{B}$ to $y_{B}$ with at least $k-s+1$ base vertices such that all edges of $\mathcal{P}_{3}$ are contained in $V(\mathcal{B})$. Similarly, we find a Berge path $\mathcal{P}_{4}$ from $v_{1}$ to $v_{2}$ with at least $s$ base vertices such that all edges of $\mathcal{P}_{4}$ are contained in $V\left(\mathcal{H}^{\prime}\right)$.

Then $\mathcal{P}_{1} \cup \mathcal{P}_{3} \cup a \cup \mathcal{P}_{2} \cup \mathcal{P}_{4}$ is a Berge cycle of length at least $(k-s+1)+s-1=k$, a contradiction.

Claim 61. If $H^{\prime}$ contains a subgraph $S$ that is s-path connected, then $H^{\prime}$ is also s-path connected.

Proof. Let $\{x, y\} \subset V\left(H^{\prime}\right)$. We will show that there exists an $(x, y)$-path in $H^{\prime}$ with at least $s$ vertices. Let $P_{x}, P_{y}$ be two disjoint shortest paths from $\{x, y\}$ to $V(S)$, say with endpoints $v_{x}$ and $v_{y}$ respectively (where possibly one or both paths are singletons). Such paths exist because $H^{\prime}$ is 2-connected. Let $P_{S}$ be a $\left(v_{x}, v_{y}\right)$-path in $S$ of length at least $S$. Then $P_{x} \cup P_{S} \cup P_{y}$ has length at least $s$.

Therefore the previous claim shows that $H^{\prime}$ is $q$-path connected. Applying Claim 60 and Theorem 26, we get

$$
e(\mathcal{B}) \leqslant N_{\mathrm{Sp}}(B, r) \leqslant \frac{|V(B)|-2}{k-q-2}\left(\begin{array}{c}
k-q \\
\min \{r,\lfloor(k-q) / 2\rfloor\}
\end{array}\right) .
$$

Summing up over all blocks deleted via big cuts, we obtain

$$
m_{B} \leqslant n_{B}\left(\frac{1}{k-q-2}\left(\begin{array}{c}
k-q \\
\min \{r,\lfloor(k-q) / 2\rfloor\}
\end{array}\right)\right)
$$

Claim 62. For each integer $s \geqslant 3, \frac{1}{s-2}\left(\begin{array}{c}s \\ \min \{r,\lfloor s / 2\rfloor\}\end{array}\right) \leqslant\left(\begin{array}{c}s \\ \min \{r-1,\lfloor s / 2\rfloor\}\end{array}\right)$.

Proof. The case for $\min \{r,\lfloor s / 2\rfloor\}=\lfloor s / 2\rfloor$ is trivial. So we may assume $s \geqslant 2 r+2$. We have $\frac{1}{s-2}\left(\begin{array}{c}s \\ r\end{array}\right)=\frac{1}{s-2} \frac{s-r+1}{r}\left(\begin{array}{c}s \\ r-1\end{array}\right) \leqslant\left(\begin{array}{c}s \\ r-1\end{array}\right)$. 
So first suppose that $\left|V\left(\mathcal{H}^{\prime}\right)\right| \geqslant k$. By Kopylov's theorem, $t+2 \leqslant q \leqslant k-2$, and $V\left(H^{\prime}\right)-V(Q)$ can be removed via $(k-s)$-disintegration. Therefore

$$
e\left(\mathcal{H}^{\prime}\right) \leqslant\left(\begin{array}{c}
q \\
\min \{r,\lfloor q / 2\rfloor\}
\end{array}\right)+\left(\left|V\left(\mathcal{H}^{\prime}\right)\right|-q\right)\left(\begin{array}{c}
k-q \\
\min \{r-1,\lfloor(k-q) / 2\rfloor\}
\end{array}\right),
$$

and hence by (16) and the previous claim,

$$
\begin{aligned}
e(\mathcal{H})=e\left(\mathcal{H}^{\prime}\right)+m_{B}+m_{S} \leqslant & \left(\begin{array}{c}
q \\
\min \{r,\lfloor q / 2\rfloor\}
\end{array}\right)+\left(\left|V\left(\mathcal{H}^{\prime}\right)\right|-q\right)\left(\begin{array}{c}
k-q \\
\min \{r-1,\lfloor(k-q) / 2\rfloor\}
\end{array}\right) \\
& +n_{B}\left(\frac{1}{k-q-2}\left(\begin{array}{c}
k-q \\
\min \{r,\lfloor(k-q) / 2\rfloor\}
\end{array}\right)\right)+n_{S} \\
\leqslant & \left(\begin{array}{c}
q \\
\min \{r,\lfloor q / 2\rfloor\}
\end{array}\right)+(n-q)\left(\begin{array}{c}
\min \{r-1,\lfloor(k-q) / 2\rfloor\} \\
\min \{r
\end{array}\right) \\
\leqslant & \max \{f(n, k, r, t), f(n, k, r, 2)\},
\end{aligned}
$$

where the last inequality follows from the convexity of the function $f$. So from now on we may assume $\left|V\left(H^{\prime}\right)\right| \leqslant k-1$.

Claim 63. Let $S$ be a 1-hamiltonian subgraph of $H^{\prime}$ with $s:=|V(S)|$ and $t+2 \leqslant s \leqslant k-2$. Let $S^{\prime}$ be the result of $(k-s)$-disintegration applied to $H^{\prime}$. Then $S^{\prime}$ is also 1-hamiltonian.

Proof. We will show a stronger statement: $S^{\prime}$ is $\left(k-\left|V\left(S^{\prime}\right)\right|\right)$-hamiltonian. Suppose not. Set $s^{\prime}:=\left|V\left(S^{\prime}\right)\right|$. Applying Theorem 25 with $d=k-s($ so $d \leqslant 2 t+2-(t+2)=t)$ and $\ell=k-s^{\prime}$, we get

$$
N_{\mathrm{Sp}}\left(S^{\prime}, r\right) \leqslant \max \left\{h_{\mathrm{Sp}}\left(s^{\prime}, k-s^{\prime}, r, k-s\right), h_{\mathrm{Sp}}\left(s^{\prime}, k-s^{\prime}, r,\left\lfloor s^{\prime} / 2\right\rfloor,\right)\right\} .
$$

If $h_{\mathrm{Sp}}\left(q^{\prime}, k-s^{\prime}, r, k-s\right) \geqslant h_{\mathrm{Sp}}\left(s^{\prime}, k-s^{\prime}, r,\left\lfloor s^{\prime} / 2\right\rfloor\right)$, then

$$
\begin{aligned}
N_{\mathrm{Sp}}\left(S^{\prime}, r\right) & \leqslant h_{\mathrm{Sp}}\left(s^{\prime}, k-s^{\prime}, r, k-s\right) \\
& =\left(\begin{array}{c}
s \\
\min \{r,\lfloor s / 2\rfloor\}
\end{array}\right)+\left(s^{\prime}-s\right)\left(\begin{array}{c}
k-s \\
\min \{r-1,\lfloor(k-s) / 2\rfloor\}
\end{array}\right) \\
& =f\left(s^{\prime}, k, r, k-s\right),
\end{aligned}
$$

Recall that since $S$ is 1-hamiltonian, $H^{\prime}$ is $s$-path connected. Hence for each $\mathcal{B}$ deleted in an application of $(\mathrm{T} 5), \partial_{2} \mathcal{B}$ is not $(k-s+1)$-path connected.

It follows that

$$
\begin{gathered}
e(\mathcal{H}) \leqslant N_{\mathrm{Sp}}\left(H^{\prime}, r\right)+m_{B}+m_{S} \\
\leqslant f\left(s^{\prime}, k, r, k-s\right)+\left(\left|V\left(H^{\prime}\right)\right|-s^{\prime}+n_{B}\right)\left(\begin{array}{c}
k-s \\
\min \{r-1,\lfloor(k-s) / 2\rfloor\}
\end{array}\right)+n_{S} \leqslant f(n, k, r, k-s) .
\end{gathered}
$$

So by the convexity of the function $f$, we are done.

Next suppose $h_{\mathrm{Sp}}\left(s^{\prime}, k-s^{\prime}, r, k-s\right) \leqslant h_{\mathrm{Sp}}\left(s^{\prime}, k-s^{\prime}, r,\left\lfloor s^{\prime} / 2\right\rfloor\right)$. For simplicity, let $a:=\left\lfloor s^{\prime} / 2\right\rfloor$. We have that $2 \leqslant a \leqslant\lfloor(k-1) / 2\rfloor=t$. 


$$
\begin{aligned}
N_{\mathrm{Sp}}\left(S^{\prime}, r\right) & \leqslant h_{\mathrm{Sp}}\left(s^{\prime}, k-s^{\prime}, r, a\right) \\
& =\left(\begin{array}{c}
s^{\prime}-\left(a-k+s^{\prime}\right) \\
\min \left\{r,\left\lfloor\left(s^{\prime}-\left(a-k+s^{\prime}\right)\right) / 2\right\rfloor\right\}
\end{array}\right)+\left(a-k+s^{\prime}\right)\left(\begin{array}{c}
a \\
k-a \\
\min \{r-1,\lfloor a / 2\rfloor\}
\end{array}\right) \\
& =\left(\begin{array}{c}
a \\
\min \{r,\lfloor(k-a) / 2\rfloor\}
\end{array}\right)+\left(s^{\prime}-(k-a)\right)\left(\begin{array}{c}
a \\
\min \{r-1,\lfloor a / 2\rfloor\}
\end{array}\right) \\
& \leqslant f\left(s^{\prime}, k, r, a\right) \leqslant f\left(s^{\prime}, k, r, t\right) .
\end{aligned}
$$

Therefore

$$
e(\mathcal{H}) \leqslant f\left(s^{\prime}, k, r, t\right)+\left(\left|V\left(H^{\prime}\right)\right|-s^{\prime}+n_{B}\right)\left(\begin{array}{c}
k-s \\
\min \{r-1,\lfloor(k-s) / 2\rfloor\}
\end{array}\right)+n_{S} \leqslant f(n, k, r, t) .
$$

Starting from the 1-hamiltonian subgraph $Q$ of $H^{\prime}$, we obtain a sequence of graphs $Q=$ $Q_{0} \subset Q_{1} \subset \ldots \subset Q_{q}$ such that $Q_{i}$ is the resulting 1-hamiltonian subgraph obtained from $\left(k-\left|V\left(Q_{i-1}\right)\right|\right)$-disintegration applied to $H^{\prime}$. The sequence ends when either the graph $Q_{q+1}$ resulting from the $\left(k-\left|V\left(Q_{q}\right)\right|\right)$-disintegration of $H^{\prime}$ is exactly $Q_{q}$, or $\left|V\left(Q_{q}\right)\right|=k-1$. In the former case, we have that $\left|V\left(Q_{q+1}\right)\right|=\left|V\left(Q_{q}\right)\right|=: q^{\prime}$. Then

$$
\begin{gathered}
e(\mathcal{H}) \leqslant N_{\mathrm{Sp}}\left(H^{\prime}, r\right)+m_{B}+m_{S} \\
\leqslant f\left(q^{\prime}, k, r, k-q^{\prime}\right)+\left(\left|V\left(H^{\prime}\right)\right|-q^{\prime}+n_{B}\right)\left(\begin{array}{c}
k-q^{\prime} \\
\min \left\{r-1,\left\lfloor\left(k-q^{\prime}\right) / 2\right\rfloor\right\}
\end{array}\right)+n_{S} \leqslant f\left(n, k, r, k-q^{\prime}\right) .
\end{gathered}
$$

Finally suppose that $\left|V\left(Q_{q}\right)\right|=k-1$. Then $H^{\prime}$ is $(k-1)$-path connected. Because $\mathcal{H}^{\prime}$ is 2-connected, we can complete a Berge path in $\mathcal{H}^{\prime}$ with at least $k-1$ vertices to a Berge cycle of length at least $k$. This proves the theorem.

\section{Proof of Theorem 17 for paths}

Proof. Let $\mathcal{H}$ be a counterexample of Theorem 17 with minimum $\sum_{e \in E(\mathcal{H})}|e|$ on at least $k+1$ vertices. If $\mathcal{H}$ contains a Berge cycle of length $k+1$ or longer, then removing any edge from this Berge cycle yields a Berge path with at least $k+1$ base vertices, a contradiction. If $\mathcal{H}$ contains a Berge cycle of length exactly $k$, then we use the following Lemma which contradicts that $n:=|V(\mathcal{H})| \geqslant k+1$.

Lemma 64 (Györi, Katona, and Lemons [11]). Let $\mathcal{H}$ be a connected hypergraph with no Berge path of length $k$. If there is a Berge cycle of length $k$ on the vertices $v_{1}, \ldots, v_{k}$ then these vertices constitute a component of $\mathcal{H}$.

Therefore $\mathcal{H}$ contains no Berge cycle of length $k$ or longer. If $\mathcal{H}$ is 2 -connected, then by Theorem 14, e(H) $\leqslant \max \{f(n, k, r, 2), f(n, k, r,\lfloor(k-1) / 2\rfloor)\}$, and we are done.

Now suppose $\mathcal{H}$ is not 2-connected. Then the incidence bigraph $I_{\mathcal{H}}$ of $\mathcal{H}$ contains a set of cut vertices. If a cut vertex $x$ of $I_{\mathcal{H}}$ corresponds to an edge in $\mathcal{H}$, then we say $x$ is a cut edge of $\mathcal{H}$. Otherwise, we say $x$ is a cut vertex of $\mathcal{H}$. 
Suppose $\mathcal{H}$ has an cut-edge $e$. We claim that for each component $\mathcal{C}$ of $\mathcal{H} \backslash e$,

$$
|V(\mathcal{C}) \cap e| \leqslant 1
$$

Indeed suppose that some component $\mathcal{C}$ of $\mathcal{H} \backslash e$ contains at least 2 vertices in $e$. Let $\mathcal{H}^{\prime}$ be the $r^{-}$-graph obtained by shrinking $e$ to remove all but one vertex in $\mathcal{C}$ from $e$. Then $\mathcal{H}^{\prime}$ is still connected and Sperner (since $e$ is a cut edge of $\mathcal{H}$ ). Furthermore, after this operation, the length of a longest path cannot increase. This contradicts the choice of $\mathcal{H}$.

Now suppose $\mathcal{H}$ contains a cut edge $e$. By (17), $e$ intersects every component of $\mathcal{H} \backslash e$ in at most one vertex. Let $\mathcal{H}^{\prime}$ be the $r^{-}$-graph obtained by contracting two vertices of $e$ into a single vertex (and then deleting $e$ if it now contains only one vertex). The new $r^{-}$-graph $\mathcal{H}^{\prime}$ is Sperner, contains no Berge $P_{k}$, and is connected. If $\left|V\left(\mathcal{H}^{\prime}\right)\right| \geqslant k+2$, we obtain that $\mathcal{H}^{\prime}$ contradicts the choice of $\mathcal{H}$ (note that $e\left(\mathcal{H}^{\prime}\right) \geqslant e(\mathcal{H})-1 \geqslant \max \{f(n, k, r, 1), f(n, k, r,\lfloor(k-$ $1) / 2\rfloor)\}-1 \geqslant \max \{f(n-1, k, r, 1), f(n-1, k, r,\lfloor(k-1) / 2\rfloor)\})$.

Iterating this process, we may assume that $\mathcal{H}$ contains no cut edges, unless $n=k+1$.

Case 1: $\mathcal{H}$ does not have a cut edge.

Since $\mathcal{H}$ is not 2 -connected, it contains at least one cut vertex. Call a subhypergraph $\mathcal{B}$ of $\mathcal{H}$ a block if the incidence bigraph of $\mathcal{B}, I_{\mathcal{B}}$, is a maximal 2-connected subgraph of $\mathcal{H}$. In particular, $\mathcal{B}$ is a Sperner 2-connected $r^{-}$-graph. Let $\mathcal{B}_{1}, \ldots, \mathcal{B}_{p}$ be the blocks of $\mathcal{H}$. For each $i$, let $s_{i}$ be the length of a longest Berge cycle in $\mathcal{B}_{i}$. Without loss of generality, we may assume $s_{1} \geqslant \ldots \geqslant s_{p}$.

Claim 65. For all $i \geqslant 2, s_{1}+s_{i} \leqslant k+1$.

In particular, $s_{i} \leqslant(k+1) / 2$ for all $i \geqslant 2$.

Proof. Suppose $s_{1}+s_{i} \geqslant k+2$. Let $C_{1}$ be a Berge cycle of $\mathcal{B}_{1}$ of length $s_{1}$ and let $C_{i}$ be a Berge cycle of $\mathcal{B}_{i}$ of length $s_{i}$. Let $P$ be a shortest Berge path from $V\left(\mathcal{B}_{1}\right)$ to $V\left(\mathcal{B}_{i}\right)$. Note that $P$ contains at most one edge from each Berge cycle. Then removing an edge from each Berge cycle, we obtain together with $P$ a Berge path whose base vertices cover $V\left(C_{1}\right) \cup V\left(C_{i}\right)$. Since $\left|V\left(C_{1}\right) \cap V\left(C_{i}\right)\right| \leqslant 1$, this path has at least $s_{1}+s_{i}-1 \geqslant k+1$ base vertices.

For each block $\mathcal{B}_{i}$, let $n_{i}:=\left|V\left(\mathcal{B}_{i}\right)\right|$. If $n_{i}=s_{i}$, then

$$
e\left(\mathcal{B}_{i}\right) \leqslant\left(\begin{array}{c}
s_{i} \\
\min \left\{r,\left\lfloor s_{i} / 2\right\rfloor\right\}
\end{array}\right) \leqslant\left(n_{i}-1\right)\left(\begin{array}{c}
s_{i}-1 \\
\min \left\{r-1,\left\lfloor\left(s_{i}-1\right) / 2\right\rfloor\right\}
\end{array}\right) .
$$

If $n_{i} \geqslant s_{i}+1$, then we apply Theorem 14 to $\mathcal{B}_{i}$ with cycle length $s_{i}+1$. We obtain

$$
e\left(\mathcal{B}_{i}\right) \leqslant \max \left\{f\left(n_{i}, s_{i}+1, r, 2\right), f\left(n_{i}, s_{i}+1,\left\lfloor s_{i} / 2\right\rfloor\right\} .\right.
$$

Furthermore, 


$$
\begin{aligned}
f\left(n_{i}, s_{i}+1, r, 2\right)= & \left(\begin{array}{c}
s_{i}-1 \\
\min \left\{r,\left\lfloor\left(s_{i}-1\right) / 2\right\rfloor\right\}
\end{array}\right)+2\left(n_{i}-s_{i}+1\right) \\
\leqslant & \left(s_{i}-1\right)\left(\begin{array}{c}
s_{i}-2 \\
\min \left\{r-1,\left\lfloor\left(s_{i}-2\right) / 2\right\rfloor\right\}
\end{array}\right) \\
& +\left(n_{i}-s_{i}\right)\left(\begin{array}{c}
s_{i}-2 \\
\min \left\{r-1,\left\lfloor\left(s_{i}-2\right) / 2\right\rfloor\right\}
\end{array}\right) \\
= & \left(n_{i}-1\right)\left(\begin{array}{c}
s_{i}-2 \\
\min \left\{r-1,\left\lfloor\left(s_{i}-2\right) / 2\right\rfloor\right\}
\end{array}\right) .
\end{aligned}
$$

And $f\left(n_{i}, s_{i}+1, r,\left\lfloor s_{i} / 2\right\rfloor\right) \leqslant\left(n_{i}-1\right)\left(\begin{array}{c}s_{i}-1 \\ \min \left\{r-1,\left\lfloor\left(s_{i}-1\right) / 2\right\rfloor\right\}\end{array}\right)$.

In all cases we get

$$
e\left(\mathcal{B}_{i}\right) \leqslant\left(n_{i}-1\right)\left(\begin{array}{c}
s_{i}-1 \\
\min \left\{r-1,\left\lfloor\left(s_{i}-1\right) / 2\right\rfloor\right\}
\end{array}\right) .
$$

For $\mathcal{B}_{1}$, if $n_{1}=s_{1}$ then $e\left(\mathcal{B}_{1}\right) \leqslant\left(\begin{array}{l}s_{1} \\ \min \left\{r,\left\lfloor s_{1} / 2\right\rfloor\right\}\end{array}\right)$ and so by $(18)$,

$$
e(\mathcal{H}) \leqslant\left(\begin{array}{c}
s_{1} \\
\min \left\{r,\left\lfloor s_{1} / 2\right\rfloor\right\}
\end{array}\right)+\sum_{i=2}^{p}\left(n_{i}-1\right)\left(\begin{array}{c}
s_{i}-1 \\
\min \left\{r-1,\left\lfloor\left(s_{i}-2\right) / 2\right\rfloor\right\}
\end{array}\right) .
$$

If $s_{1} \geqslant\lceil(k+1) / 2\rceil$, then from (19) we obtain

$$
\begin{gathered}
e(\mathcal{H}) \leqslant\left(\begin{array}{c}
s_{1} \\
\min \left\{r,\left\lfloor s_{1} / 2\right\rfloor\right\}
\end{array}\right)+\sum_{i=2}^{p}\left(n_{i}-1\right)\left(\begin{array}{c}
k-s_{1} \\
\min \left\{r-1,\left\lfloor\left(k-s_{1}\right) / 2\right\rfloor\right\}
\end{array}\right) \leqslant f\left(n, k, r, k-s_{1}\right) \\
\leqslant \max \{f(n, k, r, 1), f(n, k, r,\lfloor(k-1) / 2\rfloor\}) .
\end{gathered}
$$

Otherwise,

$$
e(\mathcal{H}) \leqslant\left(\begin{array}{c}
s_{1} \\
\min \left\{r,\left\lfloor s_{1} / 2\right\rfloor\right\}
\end{array}\right)+\sum_{i=2}^{p}\left(n_{i}-1\right)\left(\begin{array}{c}
s_{1}-1 \\
\min \left\{r-1,\left\lfloor\left(s_{1}-1\right) / 2\right\rfloor\right\}
\end{array}\right) \leqslant f(n, k, r,\lfloor(k-1) / 2\rfloor) .
$$

If $n_{1} \geqslant s_{1}+1$, then we get

$$
e\left(\mathcal{B}_{1}\right) \leqslant \max \left\{f\left(n_{1}, s_{1}+1, r, 2\right), f\left(n_{1}, s_{1}+1, r,\left\lfloor s_{1} / 2\right\rfloor\right\}\right) .
$$

If $f\left(n_{1}, s_{1}+1, r,\left\lfloor s_{1} / 2\right\rfloor\right) \geqslant f\left(n_{1}, s_{1}+1, r, 2\right)$, then together with (18), we get

$$
e(\mathcal{H}) \leqslant f\left(n_{1}, s_{1}+1, r,\left\lfloor s_{1} / 2\right\rfloor\right)+\sum_{i=2}^{p}\left(n_{i}-1\right)\left(\begin{array}{c}
\left\lfloor\frac{k-1}{2}\right\rfloor \\
\min \left\{r-1,\left\lfloor\frac{k-1}{4}\right\rfloor\right\}
\end{array}\right) \leqslant f(n, k, r,\lfloor(k-1) / 2\rfloor) .
$$


If $f\left(n_{1}, s_{1}+1, r,\left\lfloor s_{1} / 2\right\rfloor\right)<f\left(n_{1}, s_{1}+1, r, 2\right)$, then

$$
f\left(n_{1}, s_{1}+1, r, 2\right)=\left(\begin{array}{c}
s_{1}-1 \\
\min \left\{r,\left\lfloor\left(s_{1}-1\right) / 2\right\rfloor\right\}
\end{array}\right)+2\left(n_{1}-s_{1}+1\right) \leqslant\left(\begin{array}{c}
s_{1} \\
\min \left\{r,\left\lfloor s_{1} / 2\right\rfloor\right\}
\end{array}\right)+2\left(n_{1}-s_{1}\right) .
$$

Thus we obtain

$$
e(\mathcal{H}) \leqslant\left(\begin{array}{c}
s_{1} \\
\min \left\{r,\left\lfloor s_{1} / 2\right\rfloor\right\}
\end{array}\right)+2\left(n_{1}-s_{1}\right)+\sum_{i=2}^{p}\left(n_{i}-1\right)\left(\begin{array}{c}
s_{i}-1 \\
\min \left\{r-1,\left\lfloor\left(s_{i}-1\right) / 2\right\rfloor\right\}
\end{array}\right),
$$

and we are done as in the the case for (19).

Case 2: $n=k+1$ and $\mathcal{H}$ contains a cut edge.

Let $e$ be a cut edge of $\mathcal{H}$. By (17), each component $\mathcal{C}$ of $\mathcal{H} \backslash e$ contains only at most one vertex of $e$. If $|e| \geqslant 3$, then $e(\mathcal{H} \backslash e) \leqslant\left(\begin{array}{c}k+1-2 \\ \min \{r,\lfloor(k+1-2) / 2\rfloor\}\end{array}\right)$. Hence $e(\mathcal{H}) \leqslant$ $\left(\begin{array}{l}k-1 \\ \min \{r,\lfloor(k-1) / 2\rfloor\}\end{array}\right)+1<f(n, k, r, 1)$.

So we may assume $|e|=2$. Suppose first that $\mathcal{H} \backslash e$ contains a component $\mathcal{C}$ with $2 \leqslant|V(\mathcal{C})| \leqslant k-1$.

Then

$$
\begin{aligned}
e(\mathcal{H}) & \leqslant 1+\left(\begin{array}{c}
|V(\mathcal{C})| \\
\min \{r,\lfloor|V(\mathcal{C})| / 2\rfloor\}
\end{array}\right)+\left(\begin{array}{c}
(k+1)-|V(\mathcal{C})| \\
\min \{r,\lfloor((k+1)-|V(\mathcal{C})|) / 2\rfloor\}
\end{array}\right) \\
& \leqslant 1+\left(\begin{array}{c}
k-1 \\
\min \{r,\lfloor(k-1) / 2\rfloor\}
\end{array}\right)+1 \\
& =f(n, r, k, 1) .
\end{aligned}
$$

Thus $\mathcal{H} \backslash e$ must consist of one component of size $k$ and one of size 1 . The same also holds for every other cut edge $e^{\prime}$ of $\mathcal{H}$. This together with (17) implies that if $\mathcal{H}$ has two cut edges $e, e^{\prime}$, then $e^{\prime}$ is a cut edge of $\mathcal{H} \backslash e$, and vice versa. Therefore $e(\mathcal{H}) \leqslant\left(\begin{array}{c}k-1 \\ \min \{r,\lfloor(k-1) / 2\rfloor\}\end{array}\right)+2=f(n, k, r, 1)$.

So we may assume that $e$ is the only cut edge of $\mathcal{H}$. Let $\mathcal{C}$ be the component of $\mathcal{H}$ of size $k$. This component cannot contain a Berge cycle of length $k$, otherwise with $e$ we would obtain Berge path with of length $k$.

If $\mathcal{C}$ is 2 -connected, then by Theorem 14 ,

$$
e(\mathcal{H})=e(\mathcal{C})+1 \leqslant \max \{f(k, k, r, 2), f(k, k, r,\lfloor(k-1) / 2\rfloor)\}<f(n, k, r, 1) .
$$

Otherwise $\mathcal{C}$ has a cut vertex $v$ and a block $\mathcal{B}$ with $2 \leqslant|V(\mathcal{B})| \leqslant k-1$. Therefore

$$
\begin{aligned}
e(\mathcal{C}) & \leqslant\left(\begin{array}{c}
|V(\mathcal{B})| \\
\min \{r,\lfloor|V(\mathcal{B})| / 2\rfloor\}
\end{array}\right)+\left(\begin{array}{c}
k-|V(\mathcal{B})|+1 \\
\min \{r,\lfloor(k-|V(\mathcal{B})|+1) / 2\rfloor\}
\end{array}\right) \\
& \leqslant\left(\begin{array}{c}
k-1 \\
\min \{r,\lfloor(k-1) / 2\rfloor\}
\end{array}\right)+1,
\end{aligned}
$$

so we get $e(\mathcal{H})=e(\mathcal{C})+1 \leqslant f(n, k, r, 1)$. This proves the theorem. 


\section{Concluding remarks}

1. As it is mentioned in Theorem 16, if $k \geqslant 4 r$ and $n$ is asymptotically larger than $\frac{2^{r-1}}{r} k$, then our bound is also exact for $r$-graphs: a sharpness example is $\mathcal{H}_{n, k, r,\lfloor(k-1) / 2\rfloor}$. We think that for smaller $n$, our bound for $r$-graphs is not exact. It would be interesting and challenging to find exact bounds for the number of edges in $n$-vertex 2-connected $r$-graphs with no cycles of length $k$ or longer for $k>r$ and $k \leqslant n<\frac{2^{r-1}}{r} k$.

2. When $r$ is large, $k \geqslant 4 r$ and $n$ is polynomial in $k$, then $\mathcal{H}_{n, k, r, 2}$ has not much more than $\left(\begin{array}{c}k-2 \\ r\end{array}\right)$ edges. Also $\mathcal{H}_{n, k, r, 2}$ is not uniform whenever $r \geqslant 4$. The following construction of 2-connected $r$-uniform hypergraphs also has more than $\left(\begin{array}{c}k-2 \\ r\end{array}\right)$ edges in this case, although fewer edges than $\mathcal{H}_{n, k, r, 2}$ has (and it works only for $n$ such that $n-k+2$ is divisible by $r-1$ ).

Construction 66. Fix $k \geqslant 4 r \geqslant 12, s \geqslant 1, n=k-2+s(r-1)$. Define the $n$-vertex $r$-graph $F_{n, k, r, s}$ as follows. The vertex set of $F_{n, k, r, s}$ is partitioned into $s+1$ sets $A_{1}, \ldots, A_{s}, C$ such that $|C|=k-2$ and $\left|A_{i}\right|=r-1$ for all $i \in[s]$. We fix two special vertices $c_{1}, c_{2} \in C$. The edge set of $F_{n, k, r, s}$ consists of all edges contained in $C$ and of the $2(r-1)$ edges of the form $A_{i} \cup\left\{c_{j}\right\}$ for $i \in[s]$ and $j \in[2]$.

We do not currently know of any uniform hypergraphs with more edges and no Berge cycles of length $k$ or longer.

3. Note that here we use $r^{-}$-graphs to prove a bound for $r$-graphs when $k>r$ and in [15] we used $r^{+}$-graphs (i.e. hypergraphs with the lower rank at least $r$ ) in the case $k<r$.

Acknowledgment. We thank a referee for helpful comments.

\section{References}

[1] P. Erdős and T. Gallai, On maximal paths and circuits of graphs, Acta Math. Acad. Sci. Hungar. 10 (1959), 337-356.

[2] A. Davoodi, E. Győri, A. Methuku, and C. Tompkins, An Erdős-Gallai type theorem for hypergraphs, European J. Combin. 69 (2016), 159-162.

[3] H. Enomoto, Long paths and large cycles in finite graphs, J. Graph Theory 8 (1984), $287-301$.

[4] B. Ergemlidze, E. Győri, A. Methuku, N. Salia, C. Tompkins, and O. Zamora, Asymptotics for Turán numbers of cycles in 3-uniform linear hypergraphs, J. Combin. Theory Ser. A 163 (2019), 163-181.

[5] R. J. Faudree and R. H. Schelp, Ramsey type results, Infinite and Finite Sets, Colloq. Math. J. Bolyai 10, (ed. A. Hajnal et al.), North-Holland, Amsterdam, 1975, pp. $657-665$. 
[6] R. J. Faudree and R. H. Schelp, Path Ramsey numbers in multicolorings, J. Combin. Theory Ser. B 19 (1975), 150-160.

[7] Z. Füredi, A. Kostochka, and R. Luo, Avoiding long Berge cycles, J. Combin. Theory Ser. B 137 (2019), 55-64.

[8] Z. Füredi, A. Kostochka, and R. Luo, Avoiding long Berge cycles II, exact bounds for all $n,(2018)$, arXiv:1807.06119.

[9] Z. Füredi, A. Kostochka, and R. Luo, A variation of a Theorem of Pósa, Discrete Math. 342(7) (2019), 1919-1923.

[10] Z. Füredi and M. Simonovits, The history of degenerate (bipartite) extremal graph problems, Bolyai Math. Studies 25 pp. 169-264, Erdős Centennial (L. Lovász, I. Ruzsa, and V. T. Sós, Eds.) Springer, 2013. Also see: arXiv:1306.5167.

[11] E. Győri, Gy. Y. Katona, and N. Lemons, Hypergraph extensions of the Erdős-Gallai theorem, European J. Combin. 58 (2016), 238-246.

[12] E. Győri, N. Lemons, N. Salia, and O. Zamora, The Structure of Hypergraphs without long Berge cycles, (2018), arXiv:1812.10737.

[13] E. Győri, A. Methuku, N. Salia, C. Tompkins, and M. Vizer, On the maximum size of connected hypergraphs without a path of given length, Discrete Math. 341 (2018), 2602-2605.

[14] G. N. Kopylov, Maximal paths and cycles in a graph, Dokl. Akad. Nauk SSSR 234 (1977), 19-21. (English translation: Soviet Math. Dokl. 18 (1977), no. 3, 593-596.)

[15] A. Kostochka, and R. Luo, On r-uniform hypergraphs with circumference less than $r$, Discrete Appl. Math., to appear (2019).

[16] M. Lewin, On maximal circuits in directed graphs, J. Combin. Theory Ser. B. 18 (1975), 175-179.

[17] R. Luo, The maximum number of cliques in graphs without long cycles, J. Combin. Theory Ser. B. 128 (2018), 219-226.

[18] D. R. Woodall: Maximal circuits of graphs I, Acta Math. Acad. Sci. Hungar. 28 (1976), 77-80. 


\section{Appendix}

Claim 67. For fixed positive integers $n, k$, and $r$, the function

$$
f(n, k, r, a)=\left(\begin{array}{c}
k-a \\
\min \left\{r,\left\lfloor\frac{k-a}{2}\right\rfloor\right\}
\end{array}\right)+(n-k+a)\left(\begin{array}{c}
a \\
\min \{r-1,\lfloor a / 2\rfloor\}
\end{array}\right)
$$

is convex over integers $\max \{0, k-n\} \leqslant a \leqslant k$.

In particular, if we consider $f(n, k, r, a)$ over a domain of integers, say $\{c, \ldots, d\}$ where $c, d \in \mathbb{Z}, \max \{0, k-n\} \leqslant c \leqslant d \leqslant k$ then $f(n, r, k, a)$ attains its maximum at either $a=c$ or $a=d$.

Proof. Since we only consider integer values of $a$, we may view $f(n, k, r, a)$ as a sequence of numbers.

We say a sequence of real numbers $\left(f_{i}\right)_{i=u}^{v}$ is convex if $f_{i-1}+f_{i+1} \geqslant 2 f_{i}$ for all $u<i<v$.

Fact 68. Let $u<v<w$ be integers. Suppose $\left(f_{i}\right)_{i=u}^{v+1}$ and $\left(g_{i}\right)_{i=v}^{w}$ are convex sequences of real numbers such that $f_{v}=g_{v}$ and $f_{v+1}=g_{v+1}$. Then the sequence $\left(h_{i}\right)_{i=u}^{w}$ where

$$
h_{i}:= \begin{cases}f_{i}, & u \leqslant i \leqslant v+1 \\ g_{i}, & v \leqslant i \leqslant w\end{cases}
$$

is convex.

Indeed for any $u<i<w$, either $\left(h_{i-1}, h_{i}, h_{i+1}\right)=\left(f_{i-1}, f_{i}, f_{i+1}\right)$ or $\left(h_{i-1}, h_{i}, h_{i+1}\right)=$ $\left(g_{i-1}, g_{i}, g_{i+1}\right)$.

The following two facts are easy to check.

Fact 69. The sequence $\left(x_{i}\right)_{i=0}^{\infty}$ where $x_{i}:=\left(\begin{array}{c}i \\ \lfloor i / 2\rfloor\end{array}\right)$ is convex.

Fact 70. For any fixed positive integer $r$, the sequence $\left(y_{i}\right)_{i=0}^{\infty}$ where $y_{i}=\left(\begin{array}{l}i \\ r\end{array}\right)$ is convex.

By Facts 9.3-9.5, function $g_{1}(a):=\left(\begin{array}{c}k-a \\ \min \left\{\left\lfloor\frac{k-a}{2}\right\rfloor, r\right\}\end{array}\right)$ is convex for integers $0 \leqslant a \leqslant k$, and $g_{2}(a):=\left(\begin{array}{c}a \\ \min \{r-1,\lfloor a / 2\rfloor\}\end{array}\right)$ is convex for integers $a \geqslant 0$. Here we use that $g_{1}(a)=\left(\begin{array}{c}k-a \\ r\end{array}\right)$ when $a \leqslant k-2 r$ and $g_{1}(a)=\left(\begin{array}{c}k-a \\ \lfloor k-a) / 2\rfloor\end{array}\right)$ when $a \geqslant k-2 r-1$. One can show similar cut-offs for $g_{2}(a)$.

Note that $g_{2}(a)$ is non-decreasing. We also show that $(n-k+a) \cdot g_{2}(a)$ is convex for integers $a \geqslant \max \{0, k-n\}$ :

$$
\begin{aligned}
& (n-k+(a-1)) \cdot g_{2}(a-1)+(n-k+(a+1)) \cdot g_{2}(a+1) \\
= & (n-k+a) \cdot\left(g_{2}(a-1)+g_{2}(a+1)\right)-g_{2}(a-1)+g_{2}(a+1) \\
\geqslant & (n-k+a) \cdot\left(2 g_{2}(a)\right)+0 \\
= & 2(n-k+a) \cdot g_{2}(a) .
\end{aligned}
$$

Since the sum of two convex sequences is also convex, function $g_{1}(a)+(n-k+a) \cdot g_{2}(a)=$ $f(n, k, r, a)$ is also convex for integers $\max \{0, k-n\} \leqslant a \leqslant k$. This proves the claim. 